\title{
Article \\ On Some New Fractional Ostrowski- and Trapezoid-Type Inequalities for Functions of Bounded Variations with Two Variables
}

\author{
Thanin Sitthiwirattham ${ }^{1, *(D)}$, Hüseyin Budak ${ }^{2}$, Hasan Kara ${ }^{2}$, Muhammad Aamir Ali ${ }^{3, *(D)}$ \\ and Jiraporn Reunsumrit 4 \\ 1 Mathematics Department, Faculty of Science and Technology, Suan Dusit University, \\ Bangkok 10300, Thailand \\ 2 Department of Mathematics, Faculty of Science and Arts, Düzce University, 81620 Düzce, Turkey; \\ hsyn.budak@gmail.com or huseyinbudak@duzce.edu.tr (H.B.); hasan64kara@gmail.com or \\ hasan96929@ogr.duzce.edu.tr (H.K.) \\ 3 Jiangsu Key Laboratory for NSLSCS, School of Mathematical Sciences, Nanjing Normal University, \\ Nanjing 210023, China \\ 4 Department of Mathematics, Faculty of Applied Science, King Mongkut's University of Technology North \\ Bangkok, Bangkok 10800, Thailand; jiraporn.r@sci.kmutnb.ac.th \\ * Correspondence: thanin_sit@dusit.ac.th (T.S.); mahr.muhammad.aamir@gmail.com (M.A.A.)
}

check for

updates

Citation: Sitthiwirattham, T.; Budak, H.; Kara, H.; Ali, M.A.; Reunsumrit, J. On Some New Fractional Ostrowskiand Trapezoid-Type Inequalities for Functions of Bounded Variations with Two Variables. Symmetry 2021, 13,

1724. https://doi.org/

10.3390/sym13091724

Academic Editor: Nicusor Minculete

Received: 31 August 2021

Accepted: 16 September 2021

Published: 17 September 2021

Publisher's Note: MDPI stays neutral with regard to jurisdictional claims in published maps and institutional affiliations.

Copyright: (c) 2021 by the authors. Licensee MDPI, Basel, Switzerland. This article is an open access article distributed under the terms and conditions of the Creative Commons Attribution (CC BY) license (https:/ / creativecommons.org/licenses/by/ $4.0 /)$.

\begin{abstract}
In this paper, we first prove three identities for functions of bounded variations. Then, by using these equalities, we obtain several trapezoid- and Ostrowski-type inequalities via generalized fractional integrals for functions of bounded variations with two variables. Moreover, we present some results for Riemann-Liouville fractional integrals by special choice of the main results. Finally, we investigate the connections between our results and those in earlier works. Analytic inequalities of this nature and especially the techniques involved have applications in various areas in which symmetry plays a prominent role.
\end{abstract}

Keywords: trapezoid-type inequality; fractional integrals; functions of bounded variations

\section{Introduction}

One of the most important inequalities for bounded functions is the Ostrowski inequality which gives an estimate for the deviation of the values of a smooth function from its mean value. The Ostrowski inequality is stated as follows: if $\digamma:\left[\kappa_{1}, \kappa_{2}\right] \rightarrow \mathbb{R}$ is a differentiable function with a bounded derivative, then the following integral inequality

$$
\left|\digamma(\varkappa)-\frac{1}{\kappa_{2}-\kappa_{1}} \int_{\kappa_{1}}^{\kappa_{2}} \digamma(\tau) d \tau\right| \leq\left[\frac{1}{4}+\frac{\left(\varkappa-\frac{\kappa_{1}+\kappa_{2}}{2}\right)^{2}}{\left(\kappa_{2}-\kappa_{1}\right)^{2}}\right]\left(\kappa_{2}-\kappa_{1}\right)\left\|\digamma^{\prime}\right\|_{\infty}
$$

is valid for every $\varkappa \in\left[\kappa_{1}, \kappa_{2}\right]$, which was proved by Ostrowski in 1938 [1]. Here, the constant $1 / 4$ is the best possible.

In the recent years, many versions of Ostrowski-type inequalities have been proved for some kinds of function classes, such as convex functions, bounded functions, functions of bounded variation, and so on. For example, Alomari et al. established some Ostrowski-type inequalities for s-convex functions in [2]. Moreover, some papers were devoted to study on Ostrowski-type inequalities for other kinds of convexities [3-6]. On the other hand, Set first proved the fractional version of Ostrowski inequality for s-convex functions via RiemannLiouville fractional integrals [7]. Furthermore, many studies were focused on the proof of Ostrowski-type inequalities for certain fractional integral operators, such as $k$-RiemannLiouville fractional integrals [8], local fractional integrals [9], Raina fractional integrals [10], etc. (see [11-23]). Moreover, by utilizing co-ordinated convex mapping, several Ostrowski 
inequalities were presented for the Riemann integral and Riemann-Liouville fractional integrals in [24,25], respectively.

On the other hand, Dragomir extended the Ostrowski inequality for functions of bounded variation [26]. Dragomir also proved trapezoid type inequalities and midpoint type inequalities for the functions of bounded variation in [27,28], respectively. In [29], the author presented several Simpson's type inequalities for the mappings of bounded variations. In the literature, many studies were devoted to new versions of Ostrowski-type inequalities for functions of bounded variation. For some of them, please refer to [30-42]. In [43], some important inequalities for the functions of two variables with bounded variation were given and applications in the cubature formula was provided. However, there were some minor errors in the main results of the paper [43] since the Lemma 1 in the published version of [43] is inexact. Moricz has already provided the correct version of the lemma in [44]. In [43], Budak and Sarikaya presented the corrections of these results by using the lemma proved by Moricz. For other papers on inequalities for functions of two variables with bounded variation, see [45-47].

This paper aims to establish some trapezoid and Ostrowski-type inequalities for functions of bounded variations with two variables via generalized fractional integrals. The general structure of the paper consists of six sections including an introduction. The remaining part of the paper proceeds as follows: In Section 2, we first present definitions of the functions of bounded variations and total variations. We also give the definitions of generalized fractional integrals and relations between generalized fractional integrals and other type fractional integrals. In Section 3, we prove three identities for functions of bounded variations with two variables by using the Riemann-Stieltjes integral. Trapezoidand Ostrowski-type inequalities for functions of bounded variations with two variables are established in Sections 4 and 5, respectively. At the end of the paper, some conclusions and further directions of research are discussed in Section 6.

\section{Preliminaries}

In this section, we first present the definition of the functions of bounded variation (single and two variables). Then, we summarize the generalized fractional integrals and give the relations between generalized fractional integrals and the other types of fractional integrals.

\subsection{Functions of Bounded Variation with One Variable}

Definition 1 ([48]). For any partition $P: \kappa_{1}=\varkappa_{0}<\varkappa_{1}<\ldots<\varkappa_{n}=\kappa_{2}$ of $\left[\kappa_{1}, \kappa_{2}\right]$ and $\Delta \digamma\left(\varkappa_{i}\right)=\digamma\left(\varkappa_{i+1}\right)-\digamma\left(\varkappa_{i}\right)$. If the sum

$$
\sum_{i=1}^{m}\left|\Delta \digamma\left(\varkappa_{i}\right)\right|
$$

is bounded for all partitions, then the mapping $\digamma(\varkappa)$ is called of bounded variation. We assume that $\digamma$ has a bounded variation on $\left[\kappa_{1}, \kappa_{2}\right]$, and $\sum(P)$ denotes the sum $\sum_{i=1}^{n}\left|\Delta \digamma\left(\varkappa_{i}\right)\right|$ corresponding to the partition $P$ of $\left[\kappa_{1}, \kappa_{2}\right]$. The number

$$
\bigvee_{\kappa_{1}}^{\kappa_{2}}(\digamma):=\sup \left\{\sum(P): P \in P\left(\left[\kappa_{1}, \kappa_{2}\right]\right)\right\}
$$

is called the total variation of $\digamma$ on $\left[\kappa_{1}, \kappa_{2}\right]$. Here, the family of partitions of $\left[\kappa_{1}, \kappa_{2}\right]$ is denoted by $P\left(\left[\kappa_{1}, \kappa_{2}\right]\right)$.

In [26], for the functions of bounded variation, Dragomir proved the following Ostrowski inequality. 
Theorem 1. For the mapping of bounded variation $\digamma:\left[\kappa_{1}, \kappa_{2}\right] \rightarrow \mathbb{R}$ on $\left[\kappa_{1}, \kappa_{2}\right]$. The inequality

$$
\left|\frac{1}{\kappa_{2}-\kappa_{1}} \int_{\kappa_{1}}^{\kappa_{2}} \digamma(\tau) d \tau-\digamma(\varkappa)\right| \leq\left[\frac{1}{2}+\left|\frac{\varkappa-\frac{\kappa_{1}+\kappa_{2}}{2}}{\kappa_{2}-\kappa_{1}}\right|\right] \bigvee_{\kappa_{1}}^{\kappa_{2}}(\digamma)
$$

holds for all $\varkappa \in\left[\kappa_{1}, \kappa_{2}\right]$. The constant $\frac{1}{2}$ is the best possible.

\subsection{Functions of Bounded Variation with Two Variables}

Definition 2. For any set of points $\left(\varkappa_{i}, \gamma_{i}\right)(i=0,1,2, \ldots, m)$ satisfying the conditions

$$
\begin{aligned}
& \kappa_{1}=\varkappa_{0}<\varkappa_{1}<\ldots<\varkappa_{m}=\kappa_{2} \\
& \kappa_{3}=\gamma_{0}<\gamma_{1}<\ldots<\gamma_{m}=\kappa_{4}
\end{aligned}
$$

if the sum

$$
\sum_{i=1}^{m}\left|\Delta \digamma\left(\varkappa_{i}, \gamma_{i}\right)\right|
$$

is bounded for all such sets of points, then the mapping $\digamma(\varkappa, \gamma)$ is called a bounded variation (see $[49,50])$.

As a result, the definition of total variation of a function of two variables can be described as follows:

Let $\digamma$ be of bounded variation on $Q=\left[\kappa_{1}, \kappa_{2}\right] \times\left[\kappa_{3}, \kappa_{4}\right]$ and $\sum(P)$ denotes the sum $\sum_{i=1}^{n} \sum_{j=1}^{m}\left|\Delta_{11} \digamma\left(\varkappa_{i}, \gamma_{j}\right)\right|$ corresponding to the partition $P$ of $Q$. The number

$$
\bigvee_{Q}(\digamma):=\bigvee_{\kappa_{3}}^{\kappa_{4}} \bigvee_{\kappa_{1}}^{\kappa_{2}}(\digamma):=\sup \left\{\sum(P): P \in P(Q)\right\}
$$

is called the total variation of $\digamma$ on $Q$. Here, the family of partitions of $Q$ is denoted by $P(Q)$.

Lemma 1 ([44]). If $\digamma(\tau, \xi)$ continuous on the $Q=\left[\kappa_{1}, \kappa_{2}\right] \times\left[\kappa_{3}, \kappa_{4}\right]$ and $\alpha(\tau, \xi)$ is of bounded variation, then $\alpha(\tau, \xi)$ is integrable with respect to $\digamma(\tau, \xi)$ on $Q$ in the Riemann-Stieltjes integrable sense and

$$
\begin{aligned}
& \int_{\kappa_{1}}^{\kappa_{2}} \int_{\kappa_{3}}^{\kappa_{4}} \digamma(\tau, \xi) d_{\tau} d_{\xi} \alpha(\tau, \xi) \\
= & \int_{\kappa_{1}}^{\kappa_{2}} \int_{\kappa_{3}}^{\kappa_{4}} \alpha(\tau, \xi) d_{\tau} d_{\xi} \digamma(\tau, \xi)-\int_{\kappa_{1}}^{\kappa_{2}} \alpha\left(\tau, \kappa_{4}\right) d_{\tau} \digamma\left(\tau, \kappa_{4}\right)+\int_{\kappa_{1}}^{\kappa_{2}} \alpha\left(\tau, \kappa_{3}\right) d_{\tau} \digamma\left(\tau, \kappa_{3}\right) \\
& -\int_{\kappa_{3}}^{\kappa_{4}} \alpha\left(\kappa_{2}, \xi\right) d_{\xi} \digamma\left(\kappa_{2}, \xi\right)+\int_{\kappa_{3}}^{\kappa_{4}} \alpha\left(\kappa_{1}, \xi\right) d_{\xi} \digamma\left(\kappa_{1}, \xi\right) \\
& +\digamma\left(\kappa_{2}, \kappa_{4}\right) \alpha\left(\kappa_{2}, \kappa_{4}\right)-\digamma\left(\kappa_{2}, \kappa_{3}\right) \alpha\left(\kappa_{2}, \kappa_{3}\right) \\
& -\digamma\left(\kappa_{1}, \kappa_{4}\right) \alpha\left(\kappa_{1}, \kappa_{4}\right)+\digamma\left(\kappa_{1}, \kappa_{3}\right) \alpha\left(\kappa_{1}, \kappa_{3}\right) .
\end{aligned}
$$

Lemma 2 ([43]). Assume that $\rho$ is integrable with respect to $\alpha$ on $Q$ and $\alpha$ is of bounded variation on $Q$, then

$$
\left|\int_{\kappa_{3}}^{\kappa_{4}} \int_{\kappa_{1}}^{\kappa_{2}} \rho(\varkappa, \gamma) d_{\varkappa} d_{\gamma} \alpha(\varkappa, \gamma)\right| \leq \sup _{(\varkappa, \gamma) \in Q}|\rho(\varkappa, \gamma)| \bigvee_{Q}(\alpha) .
$$




\subsection{Generalized Fractional Integrals}

In this section, we summarize some fractional integrals which will be used in our main results.

Definition 3 ([51]). Let $\digamma \in L_{1}\left[\kappa_{1}, \kappa_{2}\right]$. The Riemann-Liouville fractional integrals $\mathbf{J}_{\kappa_{1}+}^{\alpha} \digamma$ and $\mathbf{J}_{\kappa_{2}-}^{\alpha} \digamma$ of order $\alpha>0$ with $\kappa_{1} \geq 0$ are defined by

$$
\mathbf{J}_{\kappa_{1}+}^{\alpha} \digamma(\varkappa)=\frac{1}{\Gamma(\alpha)} \int_{\kappa_{1}}^{\varkappa}(\varkappa-\tau)^{\alpha-1} \digamma(\tau) d \tau, \varkappa>\kappa_{1}
$$

and

$$
\mathbf{J}_{\kappa_{2}+}^{\alpha} \digamma(\varkappa)=\frac{1}{\Gamma(\alpha)} \int_{\varkappa}^{\kappa_{2}}(\tau-\varkappa)^{\alpha-1} \digamma(\tau) d \tau, \varkappa<\kappa_{2}
$$

respectively. Here, $\Gamma(\alpha)$ is the Gamma function and $\mathbf{J}_{\kappa_{1}+}^{0} \digamma(\varkappa)=\mathbf{J}_{\kappa_{2}-}^{0} \digamma(\varkappa)=\digamma(\varkappa)$.

Definition 4 ([52]). Let $\rho:\left[\kappa_{1}, \kappa_{2}\right] \rightarrow \mathbb{R}$ be a positive increasing function on $\left(\kappa_{1}, \kappa_{2}\right]$, having a continuous derivative $\rho^{\prime}(\varkappa)$ on $\left(\kappa_{1}, \kappa_{2}\right)$. The left-sides $\left(\mathcal{J}_{\kappa_{1}+; \rho}^{\alpha} \digamma(\varkappa)\right)$ and right-sides $\left(\mathcal{J}_{\kappa_{2}-; \rho}^{\alpha} \digamma(\varkappa)\right)$ fractional integral of $\digamma$ with respect to the function $\rho$ on $\left[\kappa_{1}, \kappa_{2}\right]$ of order $\alpha>0$ are defined by

$$
\mathcal{J}_{\kappa_{1}+\rho \rho}^{\alpha} \digamma(\varkappa)=\frac{1}{\Gamma(\alpha)} \int_{\kappa_{1}}^{\varkappa} \frac{\rho^{\prime}(\tau) \digamma(\tau)}{[\rho(\varkappa)-\rho(\tau)]^{1-\alpha}} d \tau, \varkappa>\kappa_{1}
$$

and

$$
\mathcal{J}_{\kappa_{2}-; \rho}^{\alpha} \digamma(\varkappa)=\frac{1}{\Gamma(\alpha)} \int_{\varkappa}^{\kappa_{2}} \frac{\rho^{\prime}(\tau) \digamma(\tau)}{[\rho(\tau)-\rho(\varkappa)]^{1-\alpha}} d \tau, \quad \varkappa<\kappa_{2}
$$

respectively.

Riemann-Liouville fractional integrals of a function with two variables can be given as follows:

Definition 5. Let $\digamma \in L_{1}\left(\Delta=\left[\kappa_{1}, \kappa_{2}\right] \times\left[\kappa_{3}, \kappa_{4}\right]\right)$. The Riemann-Liouville fractional integrals $\mathbf{J}_{\kappa_{1}+, \kappa_{3}+}^{\alpha, \beta} \digamma, \mathbf{J}_{\kappa_{1}+, \kappa_{4}-}^{\alpha, \beta} \digamma \mathbf{J}_{\kappa_{2}-, \kappa_{3}+}^{\alpha, \beta} \digamma$ and $\mathbf{J}_{\kappa_{2}-, \kappa_{4}-}^{\alpha, \beta} \digamma$ of order $\alpha, \beta>0$ with $\kappa_{1}, \kappa_{3} \geq 0$ are defined by

$$
\begin{aligned}
& \mathbf{J}_{\kappa_{1}+, \kappa_{3}+}^{\alpha, \beta} \digamma(\varkappa, \gamma)=\frac{1}{\Gamma(\alpha) \Gamma(\beta)} \int_{\kappa_{1}}^{\varkappa} \int_{\kappa_{3}}^{\gamma}(\varkappa-\tau)^{\alpha-1}(\gamma-\xi)^{\beta-1} \digamma(\tau, \xi) d \xi d \tau, \quad \varkappa>\kappa_{1}, \gamma>\kappa_{3}, \\
& \mathbf{J}_{\kappa_{1}+, \kappa_{4}-}^{\alpha, \beta} \digamma(\varkappa, \gamma)=\frac{1}{\Gamma(\alpha) \Gamma(\beta)} \int_{\kappa_{1}}^{\varkappa} \int_{\gamma}^{\kappa_{4}}(\varkappa-\tau)^{\alpha-1}(\xi-\gamma)^{\beta-1} \digamma(\tau, \xi) d \xi d \tau, \quad \varkappa>\kappa_{1}, \gamma<\kappa_{4}, \\
& \mathbf{J}_{\kappa_{2}-, \kappa_{3}+}^{\alpha, \beta} \digamma(\varkappa, \gamma)=\frac{1}{\Gamma(\alpha) \Gamma(\beta)} \int_{\varkappa}^{\kappa_{2}} \int_{\kappa_{3}}^{\gamma}(\tau-\varkappa)^{\alpha-1}(\gamma-\xi)^{\beta-1} \digamma(\tau, \xi) d \xi d \tau, \quad \varkappa<\kappa_{2}, \gamma>\kappa_{3}
\end{aligned}
$$

and

$$
\mathbf{J}_{\kappa_{2}-\kappa_{4}-}^{\alpha, \beta} \digamma(\varkappa, \gamma)=\frac{1}{\Gamma(\alpha) \Gamma(\beta)} \int_{\varkappa}^{\kappa_{2}} \int_{\gamma}^{\kappa_{4}}(\tau-\varkappa)^{\alpha-1}(\gamma-\xi)^{\beta-1} \digamma(\tau, \xi) d \xi d \tau, \quad \varkappa<\kappa_{2}, \gamma<\kappa_{4},
$$

Definition 6 ([53]). Let $\rho:\left[\kappa_{1}, \kappa_{2}\right] \rightarrow \mathbb{R}$ be a positive increasing function on $\left(\kappa_{1}, \kappa_{2}\right]$, having a continuous derivative $\rho^{\prime}(\varkappa)$ on $\left(\kappa_{1}, \kappa_{2}\right)$ and let $\omega:\left[\kappa_{3}, \kappa_{4}\right] \rightarrow \mathbb{R}$ be a positive increasing function 
on $\left(\kappa_{3}, \kappa_{4}\right]$, having a continuous derivative $\omega^{\prime}(\gamma)$ on $\left(\kappa_{3}, \kappa_{4}\right)$. If $\digamma \in L_{1}(\Delta)$, then for $\alpha, \beta>0$, the generalized fractional integral operators for functions of two variables are defined by

$$
\begin{aligned}
\mathcal{J}_{\kappa_{1}+, \kappa_{3}+; \rho, \omega}^{\alpha, \beta} \digamma(\varkappa, \gamma)= & \frac{1}{\Gamma(\alpha) \Gamma(\beta)} \int_{\kappa_{1}}^{\varkappa} \int_{\kappa_{3}}^{\gamma} \frac{\rho^{\prime}(\tau)}{[\rho(\varkappa)-\rho(\tau)]^{1-\alpha}} \frac{\omega^{\prime}(\xi)}{[\omega(\gamma)-\omega(\xi)]^{1-\beta}} \digamma(\tau, \xi) d \xi d \tau, \\
& \varkappa>\kappa_{1}, \gamma>\kappa_{3}, \\
\mathcal{J}_{\kappa_{1}+, \kappa_{4}-; \rho, \omega}^{\alpha, \beta} \digamma(\varkappa, \gamma)= & \frac{1}{\Gamma(\alpha) \Gamma(\beta)} \int_{\kappa_{1}}^{\varkappa} \int_{\gamma}^{\kappa_{4}} \frac{\rho^{\prime}(\tau)}{[\rho(\varkappa)-\rho(\tau)]^{1-\alpha}} \frac{\omega^{\prime}(\xi)}{[\omega(\xi)-\omega(\gamma)]^{1-\beta}} \digamma(\tau, \xi) d \xi d \tau, \\
& \varkappa>\kappa_{1}, \gamma<\kappa_{4}, \\
\mathcal{J}_{\kappa_{2}-, \kappa_{3}+; \rho, \omega}^{\alpha, \beta} \digamma(\varkappa, \gamma)= & \frac{1}{\Gamma(\alpha) \Gamma(\beta)} \int_{\varkappa}^{\kappa_{2}} \int_{\kappa_{3}}^{\gamma} \frac{\rho^{\prime}(\tau)}{[\rho(\tau)-\rho(\varkappa)]^{1-\alpha}} \frac{\omega^{\prime}(\xi)}{[\omega(\gamma)-\omega(\xi)]^{1-\beta}} \digamma(\tau, \xi) d \xi d \tau, \\
& \varkappa<\kappa_{2}, \gamma>\kappa_{3}
\end{aligned}
$$

and

$$
\begin{aligned}
\mathcal{J}_{\kappa_{2}-, \kappa_{4}-; \rho, \omega}^{\alpha, \beta} \digamma(\varkappa, \gamma)= & \frac{1}{\Gamma(\alpha) \Gamma(\beta)} \int_{\varkappa}^{\kappa_{2}} \int_{\gamma}^{\kappa_{4}} \frac{\rho^{\prime}(\tau)}{[\rho(\tau)-\rho(\varkappa)]^{1-\alpha}} \frac{\omega^{\prime}(\xi)}{[\mathcal{\omega}(\xi)-\mathcal{\omega}(\gamma)]^{1-\beta}} \digamma(\tau, \xi) d \xi d \tau, \\
& \varkappa<\kappa_{2}, \gamma<\kappa_{4},
\end{aligned}
$$

respectively.

By using Definition 6, well-known fractional integrals can be obtained by some special choices. For example:

1. If we choose $\alpha=\beta=1$, the operators (5)-(8) reduce to the double Riemann integral;

2. Considering $\rho(\tau)=\tau$ and $\omega(\xi)=\xi$, then the operators (5)-(8) reduce to the RiemannLiouville fractional integrals $\mathbf{J}_{\kappa_{1}+, \kappa_{3}+}^{\alpha, \beta} \digamma(\varkappa, \gamma), \mathbf{J}_{\kappa_{1}+, \kappa_{4}-}^{\alpha, \beta} \digamma(\varkappa, \gamma) \mathbf{J}_{\kappa_{2}-, \kappa_{3}+}^{\alpha, \beta} \digamma(\varkappa, \gamma)$ and $\mathbf{J}_{\mathcal{K}_{2}-, \kappa_{4}-}^{\alpha, \beta} \digamma(\varkappa, \gamma)$, respectively;

3. For $\rho(\tau)=\ln \tau$ and $\omega(\xi)=\ln \xi$, the operators (5)-(8) reduce to the Hadamard fractional integrals $I_{\kappa_{1}+, \kappa_{3}+}^{\alpha, \beta} \digamma(\varkappa, \gamma), I_{\kappa_{1}+, \kappa_{4}-}^{\alpha, \beta} \digamma(\varkappa, \gamma), I_{\kappa_{2}-, \kappa_{3}+}^{\alpha, \beta} \digamma(\varkappa, \gamma)$ and $I_{\kappa_{2}-, \kappa_{4}-}^{\alpha, \beta} \digamma(\varkappa, \gamma)$, respectively.

\section{Some Equalities for Functions of Bounded Variations with Two Variables}

Firstly, we define the following functions which will be used frequently:

$$
\begin{aligned}
& M_{\rho}^{\alpha}\left(\kappa_{1}, \varkappa\right)=\frac{\left[\rho(\varkappa)-\rho\left(\kappa_{1}\right)\right]^{\alpha}}{\Gamma(\alpha+1)}, \\
& N_{\varrho}^{\beta}\left(\kappa_{3}, \gamma\right)=\frac{\left[\omega(\gamma)-\omega\left(\kappa_{3}\right)\right]^{\beta}}{\Gamma(\beta+1)}, \\
& M_{\rho}^{\alpha}\left(\kappa_{2}, \varkappa\right)=\frac{\left[\rho\left(\kappa_{2}\right)-\rho(\varkappa)\right]^{\alpha}}{\Gamma(\alpha+1)}
\end{aligned}
$$

and

$$
N_{\omega}^{\beta}\left(\kappa_{4}, \gamma\right)=\frac{\left[\omega\left(\kappa_{4}\right)-\omega(\gamma)\right]^{\beta}}{\Gamma(\beta+1)}
$$




$$
\begin{gathered}
M_{\rho}^{\alpha}\left(\kappa_{1}, \kappa_{2} ; \varkappa\right)=\left[\rho(\varkappa)-\rho\left(\kappa_{1}\right)\right]^{\alpha}+\left[\rho\left(\kappa_{2}\right)-\rho(\varkappa)\right]^{\alpha} \\
N_{\mathscr{\omega}}^{\beta}\left(\kappa_{3}, \kappa_{4} ; \gamma\right)=\left[\omega(\gamma)-\omega\left(\kappa_{3}\right)\right]^{\beta}+\left[\omega\left(\kappa_{4}\right)-\omega(\gamma)\right]^{\beta}
\end{gathered}
$$

for $(\varkappa, \gamma) \in \Delta$. We also denote

$$
\begin{aligned}
\kappa_{1}= & M_{\rho}^{\alpha}\left(\kappa_{1}, \varkappa\right) N_{\omega}^{\beta}\left(\kappa_{3}, \gamma\right) \digamma\left(\kappa_{1}, \kappa_{3}\right)+M_{\rho}^{\alpha}\left(\kappa_{1}, \varkappa\right) N_{\omega}^{\beta}\left(\kappa_{4}, \gamma\right) \digamma\left(\kappa_{1}, \kappa_{4}\right) \\
& +M_{\rho}^{\alpha}\left(\kappa_{2}, \varkappa\right) N_{\omega}^{\beta}\left(\kappa_{3}, \gamma\right) \digamma\left(\kappa_{2}, \kappa_{3}\right)+M_{\rho}^{\alpha}\left(\kappa_{2}, \varkappa\right) N_{\omega}^{\beta}\left(\kappa_{4}, \gamma\right) \digamma\left(\kappa_{2}, \kappa_{4}\right)
\end{aligned}
$$

for $(\varkappa, \gamma) \in \Delta$.

Throughout this paper, we denote the second partial derivative $\frac{\partial^{2} \digamma}{\partial \tau \partial \xi}$ by $\digamma_{\tau \xi}$. Moreover, let $\rho:\left[\kappa_{1}, \kappa_{2}\right] \rightarrow \mathbb{R}$ be a positive increasing function on $\left(\kappa_{1}, \kappa_{2}\right]$, having a continuous derivative $\rho^{\prime}(\varkappa)$ on $\left(\kappa_{1}, \kappa_{2}\right)$ and let $\omega:\left[\kappa_{3}, \kappa_{4}\right] \rightarrow \mathbb{R}$ be a positive increasing function on $\left(\kappa_{3}, \kappa_{4}\right]$, having a continuous derivative $\omega^{\prime}(\gamma)$ on $\left(\kappa_{3}, \kappa_{4}\right)$.

Now, we are in position to prove the following identity:

Lemma 3. If $\digamma: \Delta \rightarrow \mathbb{R}$ be a mapping of bounded variation on $\Delta$, then for $\alpha, \beta>0$, we have the following equality:

$$
\begin{aligned}
& \kappa_{1}-N_{\omega}^{\beta}\left(\kappa_{3}, \gamma\right)\left[\mathcal{J}_{\kappa_{1}+; \rho}^{\alpha} \digamma\left(\varkappa, \kappa_{3}\right)+\mathcal{J}_{\kappa_{2}-; \rho}^{\alpha} \digamma\left(\varkappa, \kappa_{3}\right)\right] \\
& -N_{\omega}^{\beta}\left(\kappa_{4}, \gamma\right)\left[\mathcal{J}_{\kappa_{1}+; \rho}^{\alpha} \digamma\left(\varkappa, \kappa_{4}\right)+\mathcal{J}_{\kappa_{2}-; \rho}^{\alpha} \digamma\left(\varkappa, \kappa_{4}\right)\right] \\
& -M_{\rho}^{\alpha}\left(\kappa_{1}, \varkappa\right)\left[\mathcal{J}_{\kappa_{3}+; \omega}^{\beta} \digamma\left(\kappa_{1}, \gamma\right)+\mathcal{J}_{\kappa_{4}-; \omega}^{\beta} \digamma\left(\kappa_{1}, \gamma\right)\right] \\
& -M_{\rho}^{\alpha}\left(\kappa_{2}, \varkappa\right)\left[\mathcal{J}_{\kappa_{3}+; \omega}^{\beta} \digamma\left(\kappa_{2}, \gamma\right)+\mathcal{J}_{\kappa_{4}-; \omega}^{\beta} \digamma\left(\kappa_{2}, \gamma\right)\right]+\mathcal{J}_{\kappa_{1}+, \kappa_{3}+; \rho, \omega}^{\alpha, \beta} \digamma(\varkappa, \gamma) \\
& +\mathcal{J}_{\kappa_{1}+, \kappa_{4}-; \rho, \omega}^{\alpha, \beta} \digamma(\varkappa, \gamma)+\mathcal{J}_{\kappa_{2}-, \kappa_{3}+; \rho, \omega}^{\alpha, \beta} \digamma(\varkappa, \gamma)+\mathcal{J}_{\kappa_{2}-, \kappa_{4}-; \rho, \omega}^{\alpha, \beta} \digamma(\varkappa, \gamma) \\
= & \frac{1}{\Gamma(\alpha+1) \Gamma(\beta+1)}\left[I_{1}-I_{2}-I_{3}+I_{4}\right],
\end{aligned}
$$

where

$$
\begin{aligned}
& I_{1}=\int_{\kappa_{1}}^{\varkappa} \int_{\kappa_{3}}^{\gamma}(\rho(\varkappa)-\rho(\tau))^{\alpha}(\omega(\gamma)-\omega(\xi))^{\beta} d_{\tau} d_{\xi} \digamma(\tau, \xi), \\
& I_{2}=\int_{\kappa_{1}}^{\varkappa} \int_{\gamma}^{\kappa_{4}}(\rho(\varkappa)-\rho(\tau))^{\alpha}(\omega(\xi)-\omega(\gamma))^{\beta} d_{\tau} d_{\xi} \digamma(\tau, \xi), \\
& I_{3}=\int_{\varkappa}^{\kappa_{2}} \int_{\kappa_{3}}^{\gamma}(\rho(\tau)-\rho(\varkappa))^{\alpha}(\omega(\gamma)-\omega(\xi))^{\beta} d_{\tau} d_{\xi} \digamma(\tau, \xi), \\
& I_{4}=\int_{\varkappa}^{\kappa_{2}} \int_{\gamma}^{\kappa_{4}}(\rho(\tau)-\rho(\varkappa))^{\alpha}(\omega(\xi)-\omega(\gamma))^{\beta} d_{\tau} d_{\xi} \digamma(\tau, \xi) .
\end{aligned}
$$

Proof. By using Lemma 1, we have

$$
\begin{aligned}
I_{1} & =\int_{\kappa_{1}}^{\varkappa} \int_{\kappa_{3}}^{\gamma}(\rho(\varkappa)-\rho(\tau))^{\alpha}(\omega(\gamma)-\omega(\xi))^{\beta} d_{\tau} d_{\xi} \digamma(\tau, \xi) \\
& =\left(\omega(\gamma)-\omega\left(\kappa_{3}\right)\right)^{\beta}\left(\rho(\varkappa)-\rho\left(\kappa_{1}\right)\right)^{\alpha} \digamma\left(\kappa_{1}, \kappa_{3}\right)-\Gamma(\alpha+1)(\omega(\gamma)
\end{aligned}
$$




$$
\begin{aligned}
& \left.-\omega\left(\kappa_{3}\right)\right)^{\beta} \mathcal{J}_{\kappa_{1}+; \rho}^{\alpha} \digamma\left(\varkappa, \kappa_{3}\right)-\Gamma(\beta+1)\left(\rho(\varkappa)-\rho\left(\kappa_{1}\right)\right)^{\alpha} \mathcal{J}_{\kappa_{3}+; \omega}^{\beta} \digamma\left(\kappa_{1}, \gamma\right) \\
& +\Gamma(\alpha+1) \Gamma(\beta+1) \mathcal{J}_{\kappa_{1}+\kappa_{3}+; \rho, \omega}^{\alpha, \beta} \digamma(\varkappa, \gamma) .
\end{aligned}
$$

Similarly, we obtain

$$
\begin{aligned}
I_{2}= & -\left(\rho(\varkappa)-\rho\left(\kappa_{1}\right)\right)^{\alpha}\left(\omega\left(\kappa_{4}\right)-\omega(\gamma)\right)^{\beta} \digamma\left(\kappa_{1}, \kappa_{4}\right)+\Gamma(\alpha+1)\left(\omega\left(\kappa_{4}\right)\right. \\
& -\omega(\gamma))^{\beta} \mathcal{J}_{\kappa_{1}+; \rho}^{\alpha} \digamma\left(\varkappa, \kappa_{4}\right)+\Gamma(\beta+1)\left(\rho(\varkappa)-\rho\left(\kappa_{1}\right)\right)^{\alpha} \mathcal{J}_{\kappa_{4}-; \omega}^{\beta} \digamma\left(\kappa_{1}, \gamma\right) \\
- & \Gamma(\alpha+1) \Gamma(\beta+1) \mathcal{J}_{\kappa_{1}+\kappa_{4}-; \rho, \omega}^{\alpha, \beta} \digamma(\varkappa, \gamma), \\
I_{3}= & -\left(\rho\left(\kappa_{2}\right)-\rho(\varkappa)\right)^{\alpha}\left(\omega(\gamma)-\omega\left(\kappa_{3}\right)\right)^{\beta} \digamma\left(\kappa_{2}, \kappa_{3}\right)+\Gamma(\alpha+1)(\omega(\gamma) \\
& \left.-\omega\left(\kappa_{3}\right)\right)^{\beta} \mathcal{J}_{\kappa_{2}-; \rho}^{\alpha} \digamma\left(\varkappa, \kappa_{3}\right)+\Gamma(\beta+1)\left(\rho\left(\kappa_{2}\right)\right. \\
& -\rho(\varkappa))^{\alpha} \mathcal{J}_{\kappa_{3}+; \omega}^{\beta} \digamma\left(\kappa_{2}, \gamma\right)-\Gamma(\alpha+1) \Gamma(\beta+1) \mathcal{J}_{\kappa_{2}-\kappa_{3}+; \rho, \omega}^{\alpha, \beta} \digamma(\varkappa, \gamma)
\end{aligned}
$$

and

$$
\begin{aligned}
I_{4}= & \left(\rho\left(\kappa_{2}\right)-\rho(\varkappa)\right)^{\alpha}\left(\omega\left(\kappa_{4}\right)-\omega(\gamma)\right)^{\beta} \digamma\left(\kappa_{2}, \kappa_{4}\right)-\Gamma(\alpha+1)\left(\omega\left(\kappa_{4}\right)\right. \\
& -\omega(\gamma))^{\beta} \mathcal{J}_{\kappa_{2}-; \rho}^{\alpha} \digamma\left(\varkappa, \kappa_{4}\right)-\Gamma(\beta+1)\left(\rho\left(\kappa_{2}\right)\right. \\
& -\rho(\varkappa))^{\alpha} \mathcal{J}_{\kappa_{4}-; \omega}^{\beta} \digamma\left(\kappa_{2}, \gamma\right)+\Gamma(\alpha+1) \Gamma(\beta+1) \mathcal{J}_{\kappa_{2}-\kappa_{4}-;, \omega, \omega}^{\alpha, \beta}(\varkappa, \gamma) .
\end{aligned}
$$

By equalities (9)-(12), we establish

$$
\begin{aligned}
& \kappa_{1}-N_{\omega}^{\beta}\left(\kappa_{3}, \gamma\right)\left[\mathcal{J}_{\kappa_{1}+; \rho}^{\alpha} \digamma\left(\varkappa, \kappa_{3}\right)+\mathcal{J}_{\kappa_{2}-; \rho}^{\alpha} \digamma\left(\varkappa, \kappa_{3}\right)\right]-N_{\omega}^{\beta}\left(\kappa_{4}, \gamma\right) \\
& \times {\left[\mathcal{J}_{\kappa_{1}+; \rho}^{\alpha} \digamma\left(\varkappa, \kappa_{4}\right)+\mathcal{J}_{\kappa_{2}-; \rho}^{\alpha} \digamma\left(\varkappa, \kappa_{4}\right)\right]-M_{\rho}^{\alpha}\left(\kappa_{1}, \varkappa\right)\left[\mathcal{J}_{\kappa_{3}+; \omega}^{\beta} \digamma\left(\kappa_{1}, \gamma\right)+\mathcal{J}_{\kappa_{4}-; \omega}^{\beta} \digamma\left(\kappa_{1}, \gamma\right)\right] } \\
&-M_{\rho}^{\alpha}\left(\kappa_{2}, \varkappa\right)\left[\mathcal{J}_{\kappa_{3}+; \omega}^{\beta} \digamma\left(\kappa_{2}, \gamma\right)+\mathcal{J}_{\kappa_{4}-; \omega}^{\beta} \digamma\left(\kappa_{2}, \gamma\right)\right]+\mathcal{J}_{\kappa_{1}+, \kappa_{3}+; \rho, \omega}^{\alpha, \beta} \digamma(\varkappa, \gamma) \\
&+ \mathcal{J}_{\kappa_{1}+, \kappa_{4}-; \rho, \omega}^{\alpha, \beta} \digamma(\varkappa, \gamma)+\mathcal{J}_{\kappa_{2}-, \kappa_{3}+; \rho, \omega}^{\alpha, \beta} \digamma(\varkappa, \gamma)+\mathcal{J}_{\kappa_{2}-, \kappa_{4}-; \rho, \omega}^{\alpha, \beta} \digamma(\varkappa, \gamma) \\
&= \frac{1}{\Gamma(\alpha+1) \Gamma(\beta+1)}\left[I_{1}-I_{2}-I_{3}+I_{4}\right] \\
& \quad \text { which completes the proof. }
\end{aligned}
$$

Lemma 4. If $\digamma: \Delta \rightarrow \mathbb{R}$ be a mapping of bounded variation on $\Delta$, then for $\alpha, \beta>0$, we have the following equality

$$
\digamma(\varkappa, \gamma)-\frac{\Gamma(\alpha+1)}{M_{\rho}^{\alpha}\left(\kappa_{1}, \kappa_{2} ; \varkappa\right)}\left[\mathcal{J}_{\varkappa-; \rho}^{\alpha} \digamma\left(\kappa_{1}, \gamma\right)+\mathcal{J}_{\varkappa+; \rho}^{\alpha} \digamma\left(\kappa_{2}, \gamma\right)\right]
$$




$$
\begin{aligned}
& -\frac{\Gamma(\beta+1)}{N_{\omega}^{\beta}\left(\kappa_{3}, \kappa_{4} ; \gamma\right)}\left[\mathcal{J}_{\gamma-; \omega}^{\beta} \digamma\left(\varkappa, \kappa_{3}\right)+\mathcal{J}_{\gamma+; \omega}^{\beta} \digamma\left(\varkappa, \kappa_{4}\right)\right] \\
& +\frac{\Gamma(\alpha+1) \Gamma(\beta+1)}{M_{\rho}^{\alpha}\left(\kappa_{1}, \kappa_{2} ; \varkappa\right) N_{\omega}^{\beta}\left(\kappa_{3}, \kappa_{4} ; \gamma\right)}\left[\mathcal{J}_{\varkappa-, \gamma-; \rho, \omega}^{\alpha, \beta} \digamma\left(\kappa_{1}, \kappa_{3}\right)+\mathcal{J}_{\varkappa-, \gamma+;, \omega}^{\alpha, \beta} \digamma\left(\kappa_{1}, \kappa_{4}\right)\right. \\
& \left.+\mathcal{J}_{\varkappa+, \gamma-; \rho, \omega}^{\alpha, \beta} \digamma\left(\kappa_{2}, \kappa_{3}\right)+\mathcal{J}_{\varkappa+, \gamma+; \rho, \omega}^{\alpha, \beta} \digamma\left(\kappa_{2}, \kappa_{4}\right)\right] \\
= & \frac{1}{M_{\rho}^{\alpha}\left(\kappa_{1}, \kappa_{2} ; \varkappa\right) N_{\omega}^{\beta}\left(\kappa_{3}, \kappa_{4} ; \gamma\right)}\left[I_{5}-I_{6}-I_{7}+I_{8}\right]
\end{aligned}
$$

where

$$
\begin{aligned}
& I_{5}=\int_{\kappa_{1}}^{\varkappa} \int_{\kappa_{3}}^{\gamma}\left(\rho(\tau)-\rho\left(\kappa_{1}\right)\right)^{\alpha}\left(\omega(\xi)-\omega\left(\kappa_{3}\right)\right)^{\beta} d_{\tau} d_{\xi} \digamma(\tau, \xi), \\
& I_{6}=\int_{\kappa_{1}}^{\kappa_{\gamma}} \int_{\gamma}^{\kappa_{4}}\left(\rho(\tau)-\rho\left(\kappa_{1}\right)\right)^{\alpha}\left(\omega\left(\kappa_{4}\right)-\omega(\xi)\right)^{\beta} d_{\tau} d_{\xi} \digamma(\tau, \xi), \\
& I_{7}=\int_{\varkappa}^{\kappa_{2}} \int_{\kappa_{3}}^{\gamma}\left(\rho\left(\kappa_{2}\right)-\rho(\tau)\right)^{\alpha}\left(\omega(\xi)-\omega\left(\kappa_{3}\right)\right)^{\beta} d_{\tau} d_{\xi} \digamma(\tau, \xi)
\end{aligned}
$$

and

$$
I_{8}=\int_{\varkappa}^{\kappa_{2}} \int_{\gamma}^{\kappa_{4}}\left(\rho\left(\kappa_{2}\right)-\rho(\tau)\right)^{\alpha}\left(\omega\left(\kappa_{4}\right)-\mathfrak{\omega}(\xi)\right)^{\beta} d_{\tau} d_{\xi} \digamma(\tau, \xi) .
$$

Proof. By using Lemma 1, we have

$$
\begin{aligned}
I_{5}= & \int_{\kappa_{1}}^{\varkappa} \int_{\kappa_{3}}^{\gamma}\left(\rho(\tau)-\rho\left(\kappa_{1}\right)\right)^{\alpha}\left(\omega(\xi)-\omega\left(\kappa_{3}\right)\right)^{\beta} d_{\tau} d_{\xi} \digamma(\tau, \xi) \\
= & \left(\omega(\gamma)-\omega\left(\kappa_{3}\right)\right)^{\beta}\left(\rho(\varkappa)-\rho\left(\kappa_{1}\right)\right)^{\alpha} \digamma(\varkappa, \gamma)-\Gamma(\alpha+1)\left(\omega(\gamma)-\omega\left(\kappa_{3}\right)\right)^{\beta} \mathcal{J}_{\varkappa-; \rho}^{\alpha} \digamma\left(\kappa_{1}, \gamma\right) \\
& -\Gamma(\beta+1)\left(\rho(\varkappa)-\rho\left(\kappa_{1}\right)\right)^{\alpha} \mathcal{J}_{\gamma-; \omega}^{\beta} \digamma\left(\varkappa, \kappa_{3}\right)+\Gamma(\alpha+1) \Gamma(\beta+1) \mathcal{J}_{\varkappa-, \gamma-; \rho, \omega}^{\alpha, \beta} \digamma\left(\kappa_{1}, \kappa_{3}\right) .
\end{aligned}
$$

Similarly, we obtain

$$
\begin{aligned}
I_{6}= & \int_{\kappa_{1}}^{\varkappa} \int_{\gamma}^{\kappa_{4}}\left(\rho(\tau)-\rho\left(\kappa_{1}\right)\right)^{\alpha}\left(\omega\left(\kappa_{4}\right)-\omega(\xi)\right)^{\beta} d_{\tau} d_{\xi} \digamma(\tau, \xi) \\
= & -\left(\rho(\varkappa)-\rho\left(\kappa_{1}\right)\right)^{\alpha}\left(\omega\left(\kappa_{4}\right)-\omega(\gamma)\right)^{\beta} \digamma(\varkappa, \gamma)+\Gamma(\alpha+1)\left(\omega\left(\kappa_{4}\right)-\omega(\gamma)\right)^{\beta} \mathcal{J}_{\varkappa-; \rho}^{\alpha} \digamma\left(\kappa_{1}, \gamma\right) \\
& +\Gamma(\beta+1)\left(\rho(\varkappa)-\rho\left(\kappa_{1}\right)\right)^{\alpha} \mathcal{J}_{\gamma+; \omega}^{\beta} \digamma\left(\varkappa, \kappa_{4}\right)-\Gamma(\alpha+1) \Gamma(\beta+1) \mathcal{J}_{\varkappa-, \gamma+; \rho, \omega}^{\alpha, \beta} \digamma\left(\kappa_{1}, \kappa_{4}\right), \\
I_{7}= & \int_{\varkappa}^{\kappa_{2}} \int_{\kappa_{3}}^{\gamma}\left(\rho\left(\kappa_{2}\right)-\rho(\tau)\right)^{\alpha}\left(\omega(\xi)-\omega\left(\kappa_{3}\right)\right)^{\beta} d_{\tau} d_{\xi} \digamma(\tau, \xi) \\
= & -\left(\rho\left(\kappa_{2}\right)-\rho(\varkappa)\right)^{\alpha}\left(\omega(\gamma)-\omega\left(\kappa_{3}\right)\right)^{\beta} \digamma(\varkappa, \gamma)+\Gamma(\alpha+1)\left(\omega(\gamma)-\omega\left(\kappa_{3}\right)\right)^{\beta} \mathcal{J}_{\varkappa+; \rho}^{\alpha} \digamma\left(\kappa_{2}, \gamma\right) \\
& +\Gamma(\beta+1)\left(\rho\left(\kappa_{2}\right)-\rho(\varkappa)\right)^{\alpha} \mathcal{J}_{\gamma-; \omega}^{\beta} \digamma\left(\varkappa, \kappa_{3}\right)-\Gamma(\alpha+1) \Gamma(\beta+1) \mathcal{J}_{\varkappa+, \gamma-; \rho, \omega}^{\alpha, \beta} \digamma\left(\kappa_{2}, \kappa_{3}\right)
\end{aligned}
$$


and

$$
\begin{aligned}
I_{8}= & \int_{\varkappa}^{\kappa_{2}} \int_{\gamma}^{\kappa_{4}}\left(\rho\left(\kappa_{2}\right)-\rho(\tau)\right)^{\alpha}\left(\omega\left(\kappa_{4}\right)-\omega(\xi)\right)^{\beta} d_{\tau} d_{\xi} \digamma(\tau, \xi) \\
= & \left(\rho\left(\kappa_{2}\right)-\rho(\varkappa)\right)^{\alpha}\left(\omega\left(\kappa_{4}\right)-\omega(\gamma)\right)^{\beta} \digamma(\varkappa, \gamma)-\Gamma(\alpha+1)\left(\omega\left(\kappa_{4}\right)-\omega(\gamma)\right)^{\beta} \mathcal{J}_{\varkappa+; \rho}^{\alpha} \digamma\left(\kappa_{2}, \gamma\right) \\
& -\Gamma(\beta+1)\left(\rho\left(\kappa_{2}\right)-\rho(\varkappa)\right)^{\alpha} \mathcal{J}_{\gamma+; \omega}^{\beta} \digamma\left(\varkappa, \kappa_{4}\right)+\Gamma(\alpha+1) \Gamma(\beta+1) \mathcal{J}_{\varkappa+, \gamma+; \rho, \omega}^{\alpha, \beta} \digamma\left(\kappa_{2}, \kappa_{4}\right) .
\end{aligned}
$$

Using equalities (13)-(16), we have the desired result.

Lemma 5. If $\digamma: \Delta \rightarrow \mathbb{R}$ be a mapping of bounded variation on $\Delta$, then for $\alpha, \beta>0$, we have the following equality

$$
\begin{aligned}
& \frac{\digamma\left(\kappa_{2}, \kappa_{4}\right)+\digamma\left(\kappa_{2}, \kappa_{3}\right)+\digamma\left(\kappa_{1}, \kappa_{4}\right)+\digamma\left(\kappa_{1}, \kappa_{3}\right)}{4} \\
& -\frac{1}{4 M_{\rho}^{\alpha}\left(\kappa_{1}, \kappa_{2}\right)}\left[\mathcal{J}_{\kappa_{2}-; \rho}^{\alpha} \digamma\left(\kappa_{1}, \kappa_{4}\right)+\mathcal{J}_{\kappa_{2}-; \rho}^{\alpha} \digamma\left(\kappa_{1}, \kappa_{3}\right)+\mathcal{J}_{\kappa_{1}+; \rho}^{\alpha} \digamma\left(\kappa_{2}, \kappa_{4}\right)+\mathcal{J}_{\kappa_{1}+; \rho}^{\alpha} \digamma\left(\kappa_{2}, \kappa_{3}\right)\right] \\
& -\frac{1}{4 N_{\omega}^{\beta}\left(\kappa_{3}, \kappa_{4}\right)}\left[\mathcal{J}_{\kappa_{4}-; \omega}^{\beta} \digamma\left(\kappa_{2}, \kappa_{3}\right)+\mathcal{J}_{\kappa_{3}+; \omega}^{\beta} \digamma\left(\kappa_{2}, \kappa_{4}\right)+\mathcal{J}_{\kappa_{4}-; \omega}^{\beta} \digamma\left(\kappa_{1}, \kappa_{3}\right)+\mathcal{J}_{\kappa_{3}+; \omega}^{\beta} \digamma\left(\kappa_{1}, \kappa_{4}\right)\right] \\
& +\frac{1}{4 M_{\rho}^{\alpha}\left(\kappa_{1}, \kappa_{2}\right) N_{\omega}^{\beta}\left(\kappa_{3}, \kappa_{4}\right)}\left[\mathcal{J}_{\kappa_{2}-, \kappa_{4}-; \rho, \omega}^{\alpha, \beta} \digamma\left(\kappa_{1}, \kappa_{3}\right)+\mathcal{J}_{\kappa_{2}-, \kappa_{3}+; \rho, \omega}^{\alpha, \beta} \digamma\left(\kappa_{1}, \kappa_{4}\right)\right. \\
& \left.+\mathcal{J}_{\kappa_{1}+, \kappa_{4}-; \rho, \omega}^{\alpha, \beta} \digamma\left(\kappa_{2}, \kappa_{3}\right)+\mathcal{J}_{\kappa_{1}+, \kappa_{3}+; \rho, \omega}^{\alpha, \beta} \digamma\left(\kappa_{2}, \kappa_{4}\right)\right] \\
& =\frac{1}{4 \Gamma(\alpha+1) \Gamma(\beta+1)} \frac{1}{M_{\rho}^{\alpha}\left(\kappa_{1}, \kappa_{2}\right) N_{\omega}^{\beta}\left(\kappa_{3}, \kappa_{4}\right)} \\
& \left.\times \int_{\kappa_{1}}^{\kappa_{2}} \int_{\kappa_{3}}^{\kappa_{2}}\left[\left(\rho\left(\kappa_{2}\right)-\rho(\tau)\right)^{\alpha}-\rho(\tau)-\rho\left(\kappa_{1}\right)\right)^{\alpha}\right] \\
& \times\left[\left(\omega\left(\kappa_{4}\right)-\omega(\xi)\right)^{\beta}-\left(\omega(\xi)-\omega\left(\kappa_{3}\right)\right)^{\beta}\right] d_{\tau} d_{\xi} \digamma(\tau, \xi) .
\end{aligned}
$$

Proof. For $\varkappa=\kappa_{2}$ and $\gamma=\kappa_{4}$ in Equation (13), we have

$$
\begin{aligned}
I_{9}= & \int_{\kappa_{1}}^{\kappa_{2}} \int_{\kappa_{3}}^{\kappa_{4}}\left(\rho(\tau)-\rho\left(\kappa_{1}\right)\right)^{\alpha}\left(\omega(\xi)-\omega\left(\kappa_{3}\right)\right)^{\beta} d_{\tau} d_{\xi} \digamma(\tau, \xi) \\
= & \left(\omega\left(\kappa_{4}\right)-\omega\left(\kappa_{3}\right)\right)^{\beta}\left(\rho\left(\kappa_{2}\right)-\rho\left(\kappa_{1}\right)\right)^{\alpha} \digamma\left(\kappa_{2}, \kappa_{4}\right)-\Gamma(\alpha+1)\left(\omega\left(\kappa_{4}\right)\right. \\
& \left.-\omega\left(\kappa_{3}\right)\right)^{\beta} \mathcal{J}_{\kappa_{2}-; \rho}^{\alpha} \digamma\left(\kappa_{1}, \kappa_{4}\right)-\Gamma(\beta+1)\left(\rho\left(\kappa_{2}\right)-\rho\left(\kappa_{1}\right)\right)^{\alpha} \mathcal{J}_{\kappa_{4}-; \omega}^{\beta} \digamma\left(\kappa_{2}, \kappa_{3}\right) \\
& +\Gamma(\alpha+1) \Gamma(\beta+1) \mathcal{J}_{\kappa_{2}-, \kappa_{4}-; \rho, \omega}^{\alpha} \digamma\left(\kappa_{1}, \kappa_{3}\right) .
\end{aligned}
$$

For $\varkappa=\kappa_{2}$ and $\gamma=\kappa_{3}$ in Equation (14), we have

$$
\begin{aligned}
I_{10}= & \int_{\kappa_{1}}^{\kappa_{2}} \int_{\kappa_{3}}^{\kappa_{4}}\left(\rho(\tau)-\rho\left(\kappa_{1}\right)\right)^{\alpha}\left(\omega\left(\kappa_{4}\right)-\omega(\xi)\right)^{\beta} d_{\tau} d_{\xi} \digamma(\tau, \xi) \\
& -\left(\rho\left(\kappa_{2}\right)-\rho\left(\kappa_{1}\right)\right)^{\alpha}\left(\omega\left(\kappa_{4}\right)-\omega\left(\kappa_{3}\right)\right)^{\beta} \digamma\left(\kappa_{2}, \kappa_{3}\right)
\end{aligned}
$$




$$
\begin{aligned}
& +\Gamma(\alpha+1)\left(\omega\left(\kappa_{4}\right)-\omega\left(\kappa_{3}\right)\right)^{\beta} \mathcal{J}_{\kappa_{2}-; \rho}^{\alpha} \digamma\left(\kappa_{1}, \kappa_{3}\right) \\
& +\Gamma(\beta+1)\left(\rho\left(\kappa_{2}\right)-\rho\left(\kappa_{1}\right)\right)^{\alpha} \mathcal{J}_{\kappa_{3}+; \omega}^{\beta} \digamma\left(\kappa_{2}, \kappa_{4}\right) \\
& -\Gamma(\alpha+1) \Gamma(\beta+1) \mathcal{J}_{\kappa_{2}-, \kappa_{3}+; \rho, \omega}^{\alpha, \beta} \digamma\left(\kappa_{1}, \kappa_{4}\right),
\end{aligned}
$$

for $\varkappa=\kappa_{1}$ and $\gamma=\kappa_{4}$ in Equation (15), we have

$$
\begin{aligned}
I_{11}= & \int_{\kappa_{1}}^{\kappa_{2}} \int_{\kappa_{3}}^{\kappa_{4}}\left(\rho\left(\kappa_{2}\right)-\rho(\tau)\right)^{\alpha}\left(\omega(\xi)-\omega\left(\kappa_{3}\right)\right)^{\beta} d_{\tau} d_{\xi} \digamma(\tau, \xi) \\
= & -\left(\rho\left(\kappa_{2}\right)-\rho\left(\kappa_{1}\right)\right)^{\alpha}\left(\omega\left(\kappa_{4}\right)-\omega\left(\kappa_{3}\right)\right)^{\beta} \digamma\left(\kappa_{1}, \kappa_{4}\right) \\
& +\Gamma(\alpha+1)\left(\omega\left(\kappa_{4}\right)-\omega\left(\kappa_{3}\right)\right)^{\beta} \mathcal{J}_{\kappa_{1}+; \rho}^{\alpha} \digamma\left(\kappa_{2}, \kappa_{4}\right) \\
& +\Gamma(\beta+1)\left(\rho\left(\kappa_{2}\right)-\rho\left(\kappa_{1}\right)\right)^{\alpha} \mathcal{J}_{\kappa_{4}-; \omega}^{\beta} \digamma\left(\kappa_{1}, \kappa_{3}\right) \\
& -\Gamma(\alpha+1) \Gamma(\beta+1) \mathcal{J}_{\kappa_{1}+, \kappa_{4}-; \rho, \omega}^{\alpha, \beta}\left(\kappa_{2}, \kappa_{3}\right)
\end{aligned}
$$

and finally, for $\varkappa=\kappa_{1}$ and $\gamma=\kappa_{3}$ in Equation (16), we find

$$
\begin{aligned}
I_{12}= & \int_{\kappa_{1}}^{\kappa_{2}} \int_{\kappa_{3}}^{\kappa_{4}}\left(\rho\left(\kappa_{2}\right)-\rho(\tau)\right)^{\alpha}\left(\omega\left(\kappa_{4}\right)-\omega(\xi)\right)^{\beta} d_{\tau} d_{\xi} \digamma(\tau, \xi) \\
= & \left(\rho\left(\kappa_{2}\right)-\rho\left(\kappa_{1}\right)\right)^{\alpha}\left(\omega\left(\kappa_{4}\right)-\omega\left(\kappa_{3}\right)\right)^{\beta} \digamma\left(\kappa_{1}, \kappa_{3}\right) \\
& -\Gamma(\alpha+1)\left(\omega\left(\kappa_{4}\right)-\omega\left(\kappa_{3}\right)\right)^{\beta} \mathcal{J}_{\kappa_{1}+; \rho}^{\alpha} \digamma\left(\kappa_{2}, \kappa_{3}\right) \\
& -\Gamma(\beta+1)\left(\rho\left(\kappa_{2}\right)-\rho\left(\kappa_{1}\right)\right)^{\alpha} \mathcal{J}_{\kappa_{3}+; \omega}^{\beta} \digamma\left(\kappa_{1}, \kappa_{4}\right) \\
& +\Gamma(\alpha+1) \Gamma(\beta+1) \mathcal{J}_{\kappa_{1}+, \kappa_{3}+; \rho, \omega}^{\alpha, \beta}\left(\kappa_{2}, \kappa_{4}\right) .
\end{aligned}
$$

Using equalities (18)-(21), we establish

$$
\begin{aligned}
& \frac{1}{4 \Gamma(\alpha+1) \Gamma(\beta+1)} \frac{1}{M_{\rho}^{\alpha}\left(\kappa_{1}, \kappa_{2}\right) N_{\omega}^{\beta}\left(\kappa_{3}, \kappa_{4}\right)}\left[I_{9}-I_{10}-I_{11}+I_{12}\right] \\
= & \frac{\digamma\left(\kappa_{2}, \kappa_{4}\right)+\digamma\left(\kappa_{2}, \kappa_{3}\right)+\digamma\left(\kappa_{1}, \kappa_{4}\right)+\digamma\left(\kappa_{1}, \kappa_{3}\right)}{4} \\
& -\frac{1}{4 M_{\rho}^{\alpha}\left(\kappa_{1}, \kappa_{2}\right)}\left[\mathcal{J}_{\kappa_{2}-; \rho}^{\alpha} \digamma\left(\kappa_{1}, \kappa_{4}\right)+\mathcal{J}_{\kappa_{2}-; \rho}^{\alpha} \digamma\left(\kappa_{1}, \kappa_{3}\right)+\mathcal{J}_{\kappa_{1}+; \rho}^{\alpha} \digamma\left(\kappa_{2}, \kappa_{4}\right)+\mathcal{J}_{\kappa_{1}+; \rho}^{\alpha} \digamma\left(\kappa_{2}, \kappa_{3}\right)\right] \\
& -\frac{1}{4 N_{\omega}^{\beta}\left(\kappa_{3}, \kappa_{4}\right)}\left[\mathcal{J}_{\kappa_{4}-; \omega}^{\beta} \digamma\left(\kappa_{2}, \kappa_{3}\right)+\mathcal{J}_{\kappa_{3}+; \omega}^{\beta} \digamma\left(\kappa_{2}, \kappa_{4}\right)+\mathcal{J}_{\kappa_{4}-; \omega}^{\beta} \digamma\left(\kappa_{1}, \kappa_{3}\right)+\mathcal{J}_{\kappa_{3}+; \omega}^{\beta} \digamma\left(\kappa_{1}, \kappa_{4}\right)\right] \\
& +\frac{1}{4 M_{\rho}^{\alpha}\left(\kappa_{1}, \kappa_{2}\right) N_{\omega}^{\beta}\left(\kappa_{3}, \kappa_{4}\right)}\left[\mathcal{J}_{\kappa_{2}-, \kappa_{4}-; \rho, \omega}^{\alpha, \beta} \digamma\left(\kappa_{1}, \kappa_{3}\right)+\mathcal{J}_{\kappa_{2}-, \kappa_{3}+; \rho, \omega}^{\alpha, \beta} \digamma\left(\kappa_{1}, \kappa_{4}\right)\right. \\
& \left.+\mathcal{J}_{\kappa_{1}+, \kappa_{4}-; \rho, \omega}^{\alpha, \beta} \digamma\left(\kappa_{2}, \kappa_{3}\right)+\mathcal{J}_{\kappa_{1}+, \kappa_{3}+; \rho, \omega}^{\alpha, \beta} \digamma\left(\kappa_{2}, \kappa_{4}\right)\right]
\end{aligned}
$$


which completes the proof.

\section{Trapezoid-Type Inequalities for Functions of Bounded Variations with Two Variables}

In this section, we present some trapezoid-type inequalities for generalized fractional integrals.

Theorem 2. If $\digamma: \Delta \rightarrow \mathbb{R}$ be a mapping of bounded variation on $\Delta$, then for $\alpha, \beta>0$, we have the following inequality

$$
\begin{aligned}
& \mid \kappa_{1}-N_{\omega}^{\beta}\left(\kappa_{3}, \gamma\right)\left[\mathcal{J}_{\kappa_{1}+; \rho}^{\alpha} \digamma\left(\varkappa, \kappa_{3}\right)+\mathcal{J}_{\kappa_{2}-; \rho}^{\alpha} \digamma\left(\varkappa, \kappa_{3}\right)\right] \\
& -N_{\omega}^{\beta}\left(\kappa_{4}, \gamma\right)\left[\mathcal{J}_{\kappa_{1}+; \rho}^{\alpha} \digamma\left(\varkappa, \kappa_{4}\right)+\mathcal{J}_{\kappa_{2}-; \rho}^{\alpha} \digamma\left(\varkappa, \kappa_{4}\right)\right] \\
& -M_{\rho}^{\alpha}\left(\kappa_{1}, \varkappa\right)\left[\mathcal{J}_{\kappa_{3}+\infty \omega}^{\beta} \digamma\left(\kappa_{1}, \gamma\right)+\mathcal{J}_{\kappa_{4}-; \omega}^{\beta} \digamma\left(\kappa_{1}, \gamma\right)\right] \\
& -M_{\rho}^{\alpha}\left(\kappa_{2}, \varkappa\right)\left[\mathcal{J}_{\kappa_{3}+; \omega}^{\beta} \digamma\left(\kappa_{2}, \gamma\right)+\mathcal{J}_{\kappa_{4}-; \omega}^{\beta} \digamma\left(\kappa_{2}, \gamma\right)\right]+\mathcal{J}_{\kappa_{1}+, \kappa_{3}+; \rho, \omega}^{\alpha, \beta} \digamma(\varkappa, \gamma) \\
& +\mathcal{J}_{\kappa_{1}+, \kappa_{4}-; \rho, \omega}^{\alpha, \beta} \digamma(\varkappa, \gamma)+\mathcal{J}_{\kappa_{2}-, \kappa_{3}+; \rho, \omega}^{\alpha, \beta} \digamma(\varkappa, \gamma)+\mathcal{J}_{\kappa_{2}-, \kappa_{4}-; \rho, \omega}^{\alpha, \beta} \digamma(\varkappa, \gamma) \mid \\
& \leq \frac{1}{\Gamma(\alpha+1) \Gamma(\beta+1)}\left[\left(\rho(\varkappa)-\rho\left(\kappa_{1}\right)\right)^{\alpha}\left(\omega(\gamma)-\omega\left(\kappa_{3}\right)\right)^{\beta} \bigvee_{\kappa_{1}}^{\varkappa} \bigvee_{\kappa_{3}}^{\gamma}(\digamma)\right. \\
& +\left(\rho(\varkappa)-\rho\left(\kappa_{1}\right)\right)^{\alpha}\left(\omega\left(\kappa_{4}\right)-\omega(\gamma)\right)^{\beta} \bigvee_{\kappa_{1}}^{\varkappa} \bigvee_{\gamma}^{\kappa_{4}}(\digamma) \\
& +\left(\rho\left(\kappa_{2}\right)-\rho(\varkappa)\right)^{\alpha}\left(\omega(\gamma)-\omega\left(\kappa_{3}\right)\right)^{\beta} \bigvee_{\varkappa}^{\kappa_{2}} \bigvee_{\kappa_{3}}^{\gamma}(\digamma) \\
& \left.+\left(\rho\left(\kappa_{2}\right)-\rho(\varkappa)\right)^{\alpha}\left(\omega\left(\kappa_{4}\right)-\omega(\gamma)\right)^{\beta} \bigvee_{\varkappa}^{\kappa_{2}} \bigvee_{\gamma}^{\kappa_{4}}(\digamma)\right] \\
& \leq \frac{1}{\Gamma(\alpha+1) \Gamma(\beta+1)}\left[\frac{1}{2}\left(\rho\left(\kappa_{2}\right)-\rho\left(\kappa_{1}\right)\right)+\left|\rho(\varkappa)-\frac{\rho\left(\kappa_{1}\right)+\rho\left(\kappa_{2}\right)}{2}\right|\right]^{\alpha} \\
& \times\left[\frac{1}{2}\left(\omega\left(\kappa_{4}\right)-\omega\left(\kappa_{3}\right)\right)+\left|\omega(\gamma)-\frac{\omega\left(\kappa_{3}\right)+\omega\left(\kappa_{4}\right)}{2}\right|\right] \bigvee_{\kappa_{1}}^{\beta} \bigvee_{\kappa_{2}}^{\kappa_{2}}(\digamma) \text {. }
\end{aligned}
$$

Proof. By taking the modulus in Lemma 3, we obtain

$$
\begin{aligned}
& \mid \kappa_{1}-N_{\omega}^{\beta}\left(\kappa_{3}, \gamma\right)\left[\mathcal{J}_{\kappa_{1}+\rho \rho}^{\alpha} \digamma\left(\varkappa, \kappa_{3}\right)+\mathcal{J}_{\kappa_{2}-; \rho}^{\alpha} \digamma\left(\varkappa, \kappa_{3}\right)\right] \\
& -N_{\omega}^{\beta}\left(\kappa_{4}, \gamma\right)\left[\mathcal{J}_{\kappa_{1}+; \rho}^{\alpha} \digamma\left(\varkappa, \kappa_{4}\right)+\mathcal{J}_{\kappa_{2}-; \rho}^{\alpha} \digamma\left(\varkappa, \kappa_{4}\right)\right] \\
& -M_{\rho}^{\alpha}\left(\kappa_{1}, \varkappa\right)\left[\mathcal{J}_{\kappa_{3}+; \omega}^{\beta} \digamma\left(\kappa_{1}, \gamma\right)+\mathcal{J}_{\kappa_{4}-; \omega}^{\beta} \digamma\left(\kappa_{1}, \gamma\right)\right] \\
& -M_{\rho}^{\alpha}\left(\kappa_{2}, \varkappa\right)\left[\mathcal{J}_{\kappa_{3}+; \omega}^{\beta} \digamma\left(\kappa_{2}, \gamma\right)+\mathcal{J}_{\kappa_{4}-; \omega}^{\beta} \digamma\left(\kappa_{2}, \gamma\right)\right]+\mathcal{J}_{\kappa_{1}+, \kappa_{3}+; \rho, \omega}^{\alpha, \beta} \digamma(\varkappa, \gamma) \\
& +\mathcal{J}_{\kappa_{1}+, \kappa_{4}-; \rho, \omega}^{\alpha, \beta} \digamma(\varkappa, \gamma)+\mathcal{J}_{\kappa_{2}-, \kappa_{3}+; \rho, \omega}^{\alpha, \beta} \digamma(\varkappa, \gamma)+\mathcal{J}_{\kappa_{2}-, \kappa_{4}-; \rho, \omega}^{\alpha, \beta} \digamma(\varkappa, \gamma) \mid
\end{aligned}
$$




$$
\begin{aligned}
\leq & \frac{1}{\Gamma(\alpha+1) \Gamma(\beta+1)}\left[\left|\int_{\kappa_{1}}^{\varkappa} \int_{\kappa_{3}}^{\gamma}(\rho(\varkappa)-\rho(\tau))^{\alpha}(\omega(\gamma)-\omega(\xi))^{\beta} d_{\tau} d_{\xi} \digamma(\tau, \xi)\right|\right. \\
& +\left|\int_{\kappa_{1}}^{\varkappa} \int_{\gamma}^{\kappa_{4}}(\rho(\varkappa)-\rho(\tau))^{\alpha}(\omega(\xi)-\omega(\gamma))^{\beta} d_{\tau} d_{\xi} \digamma(\tau, \xi)\right| \\
& +\left|\int_{\varkappa}^{\kappa_{2}} \int_{\kappa_{3}}^{\gamma}(\rho(\tau)-\rho(\varkappa))^{\alpha}(\omega(\gamma)-\omega(\xi))^{\beta} d_{\tau} d_{\xi} \digamma(\tau, \xi)\right| \\
& \left.+\left|\int_{\varkappa}^{\kappa_{2}} \int_{\gamma}^{\kappa_{4}}(\rho(\tau)-\rho(\varkappa))^{\alpha}(\omega(\xi)-\omega(\gamma))^{\beta} d_{\tau} d_{\xi} \digamma(\tau, \xi)\right|\right] .
\end{aligned}
$$

By Lemma, it follows that

$$
\begin{aligned}
& \left|\int_{\kappa_{1}}^{\varkappa} \int_{\kappa_{3}}^{\gamma}(\rho(\varkappa)-\rho(\tau))^{\alpha}(\omega(\gamma)-\omega(\xi))^{\beta} d_{\tau} d_{\xi} \digamma(\tau, \xi)\right| \\
\leq & \sup _{(\tau, \xi) \in\left[\kappa_{1}, \varkappa\right] \times\left[\kappa_{3}, \gamma\right]}\left|(\rho(\varkappa)-\rho(\tau))^{\alpha}(\omega(\gamma)-\omega(\xi))^{\beta}\right| \bigvee_{\kappa_{1}}^{\varkappa} \bigvee_{\kappa_{3}}^{\gamma}(\digamma) \\
= & \left(\rho(\varkappa)-\rho\left(\kappa_{1}\right)\right)^{\alpha}\left(\omega(\gamma)-\omega\left(\kappa_{3}\right)\right)^{\beta} \bigvee_{\kappa_{1}}^{\varkappa} \bigvee_{\kappa_{3}}^{\gamma}(\digamma) .
\end{aligned}
$$

Similarly, we obtain

$$
\begin{aligned}
& \left|\int_{\kappa_{1}}^{\varkappa} \int_{\gamma}^{\kappa_{4}}(\rho(\varkappa)-\rho(\tau))^{\alpha}(\omega(\xi)-\omega(\gamma))^{\beta} d_{\tau} d_{\xi} \digamma(\tau, \xi)\right| \\
& \leq \quad\left(\rho(\varkappa)-\rho\left(\kappa_{1}\right)\right)^{\alpha}\left(\omega\left(\kappa_{4}\right)-\omega(\gamma)\right)^{\beta} \bigvee_{\kappa_{1}}^{\varkappa} \bigvee_{\gamma}^{\kappa_{4}}(\digamma) \\
& \leq\left|\int_{\varkappa}^{\kappa_{2}} \int_{\kappa_{3}}^{\gamma}(\rho(\tau)-\rho(\varkappa))^{\alpha}(\omega(\gamma)-\omega(\xi))^{\beta} d_{\tau} d_{\xi} \digamma(\tau, \xi)\right| \\
& \leq \quad\left(\rho\left(\kappa_{2}\right)-\rho(\varkappa)\right)^{\alpha}\left(\omega(\gamma)-\omega\left(\kappa_{3}\right)\right)^{\beta} \bigvee_{\varkappa}^{\kappa_{2}} \bigvee_{\kappa_{3}}^{\gamma}(\digamma)
\end{aligned}
$$

and

$$
\begin{aligned}
& \left|\int_{\varkappa}^{\kappa_{2}} \int_{\gamma}^{\kappa_{4}}(\rho(\tau)-\rho(\varkappa))^{\alpha}(\omega(\xi)-\omega(\gamma))^{\beta} d_{\tau} d_{\xi} \digamma(\tau, \xi)\right| \\
& \leq \quad\left(\rho\left(\kappa_{2}\right)-\rho(\varkappa)\right)^{\alpha}\left(\omega\left(\kappa_{4}\right)-\omega(\gamma)\right)^{\beta} \bigvee_{\varkappa}^{\kappa_{2}} \bigvee_{\gamma}^{\kappa_{4}}(\digamma) .
\end{aligned}
$$

By substituting the inequalities (24)-(27) in Equation (23), we obtain

$$
\begin{aligned}
& \mid \kappa_{1}-N_{\omega}^{\beta}\left(\kappa_{3}, \gamma\right)\left[\mathcal{J}_{\kappa_{1}+; \rho}^{\alpha} \digamma\left(\varkappa, \kappa_{3}\right)+\mathcal{J}_{\kappa_{2}-; \rho}^{\alpha} \digamma\left(\varkappa, \kappa_{3}\right)\right] \\
& -N_{\varrho}^{\beta}\left(\kappa_{4}, \gamma\right)\left[\mathcal{J}_{\kappa_{1}+; \rho}^{\alpha} \digamma\left(\varkappa, \kappa_{4}\right)+\mathcal{J}_{\kappa_{2}-; \rho}^{\alpha} \digamma\left(\varkappa, \kappa_{4}\right)\right]
\end{aligned}
$$




$$
\begin{aligned}
& -M_{\rho}^{\alpha}\left(\kappa_{1}, \varkappa\right)\left[\mathcal{J}_{\kappa_{3}+; \omega}^{\beta} \digamma\left(\kappa_{1}, \gamma\right)+\mathcal{J}_{\kappa_{4}-; \omega}^{\beta} \digamma\left(\kappa_{1}, \gamma\right)\right] \\
& -M_{\rho}^{\alpha}\left(\kappa_{2}, \varkappa\right)\left[\mathcal{J}_{\kappa_{3}+; \omega}^{\beta} \digamma\left(\kappa_{2}, \gamma\right)+\mathcal{J}_{\kappa_{4}-; \omega}^{\beta} \digamma\left(\kappa_{2}, \gamma\right)\right]+\mathcal{J}_{\kappa_{1}+, \kappa_{3}+;, \omega}^{\alpha, \beta} \digamma(\varkappa, \gamma) \\
& +\mathcal{J}_{\kappa_{1}+, \kappa_{4}-; \rho, \omega}^{\alpha, \beta} \digamma(\varkappa, \gamma)+\mathcal{J}_{\kappa_{2}-, \kappa_{3}+; \rho, \omega}^{\alpha, \beta} \digamma(\varkappa, \gamma)+\mathcal{J}_{\kappa_{2}-, \kappa_{4}-; \rho, \omega}^{\alpha, \beta} \digamma(\varkappa, \gamma) \mid \\
\leq & \frac{1}{\Gamma(\alpha+1) \Gamma(\beta+1)}\left[\left(\rho(\varkappa)-\rho\left(\kappa_{1}\right)\right)^{\alpha}\left(\omega(\gamma)-\omega\left(\kappa_{3}\right)\right)^{\beta} \bigvee_{\kappa_{1}}^{\varkappa} \bigvee_{\kappa_{3}}^{\gamma}(\digamma)\right. \\
& +\left(\rho(\varkappa)-\rho\left(\kappa_{1}\right)\right)^{\alpha}\left(\omega\left(\kappa_{4}\right)-\omega(\gamma)\right)^{\beta} \bigvee_{\kappa_{1}}^{\varkappa} \bigvee_{\gamma}^{\kappa_{4}}(\digamma) \\
& +\left(\rho\left(\kappa_{2}\right)-\rho(\varkappa)\right)^{\alpha}\left(\omega(\gamma)-\omega\left(\kappa_{3}\right)\right)^{\beta} \bigvee_{\varkappa}^{\kappa_{2}} \bigvee_{\kappa_{3}}^{\gamma}(\digamma) \\
& \left.+\left(\rho\left(\kappa_{2}\right)-\rho(\varkappa)\right)^{\alpha}\left(\omega\left(\kappa_{4}\right)-\omega(\gamma)\right)^{\beta} \bigvee_{\varkappa}^{\kappa_{2}} \bigvee_{\gamma}^{\kappa_{4}}(\digamma)\right] \\
= & : \mathrm{Y}(\varkappa, \gamma)
\end{aligned}
$$

which gives the first inequality in Equation (22).

By the properties of maximum, we obtain

$$
\begin{aligned}
& Y(\varkappa, \gamma) \\
\leq & \max \left\{\left(\rho(\varkappa)-\rho\left(\kappa_{1}\right)\right)^{\alpha}\left(\omega(\gamma)-\omega\left(\kappa_{3}\right)\right)^{\beta},\left(\rho(\varkappa)-\rho\left(\kappa_{1}\right)\right)^{\alpha}\left(\omega\left(\kappa_{4}\right)-\omega(\gamma)\right)^{\beta},\right. \\
& \left.\left(\rho\left(\kappa_{2}\right)-\rho(\varkappa)\right)^{\alpha}\left(\omega(\gamma)-\omega\left(\kappa_{3}\right)\right)^{\beta},\left(\rho\left(\kappa_{2}\right)-\rho(\varkappa)\right)^{\alpha}\left(\omega\left(\kappa_{4}\right)-\omega(\gamma)\right)^{\beta}\right\} \\
& \times\left[\bigvee_{\kappa_{1}}^{\varkappa} \bigvee_{\kappa_{3}}^{\gamma}(\digamma)+\bigvee_{\kappa_{1}}^{\varkappa} \bigvee_{\gamma}^{\kappa_{4}}(\digamma)+\bigvee_{\varkappa}^{\kappa_{2}} \bigvee_{\kappa_{3}}^{\gamma}(\digamma)+\bigvee_{\varkappa} \bigvee_{\gamma}^{\kappa_{2}}(\digamma)\right] \\
= & \max \left\{\left(\rho(\varkappa)-\rho\left(\kappa_{1}\right)\right)^{\alpha},\left(\rho\left(\kappa_{2}\right)-\rho(\varkappa)\right)^{\alpha}\right\} \max \left\{\left(\omega(\gamma)-\omega\left(\kappa_{3}\right)\right)^{\beta},\left(\omega\left(\kappa_{4}\right)-\omega(\gamma)\right)^{\beta}\right\} \\
& \times \bigvee_{\kappa_{1}}^{\kappa_{2}} \bigvee_{\kappa_{2}}^{\kappa_{4}}(\digamma) \\
= & {\left[\frac{1}{2}\left(\rho\left(\kappa_{2}\right)-\rho\left(\kappa_{1}\right)\right)+\left|\rho(\varkappa)-\frac{\rho\left(\kappa_{1}\right)+\rho\left(\kappa_{2}\right)}{2}\right|\right]^{\alpha} } \\
& \times\left[\frac{1}{2}\left(\omega\left(\kappa_{4}\right)-\omega\left(\kappa_{3}\right)\right)+\left|\omega(\gamma)-\frac{\omega\left(\kappa_{3}\right)+\omega\left(\kappa_{4}\right)}{2}\right|\right]^{\beta} \bigvee_{\kappa_{1}} \bigvee_{\kappa_{2}}^{\kappa_{2}}(\digamma) .
\end{aligned}
$$

This completes the proof.

Corollary 1. If we take $\rho(\tau)=\tau, \tau \in\left[\kappa_{1}, \kappa_{2}\right]$ and $\mathfrak{\omega}(\xi)=\xi, \xi \in\left[\kappa_{3}, \kappa_{4}\right]$ in Theorem 2, then we have the following trapezoid-type inequalities for Riemann-Liouville fractional integrals

$$
\mid \frac{\left(\varkappa-\kappa_{1}\right)^{\alpha}\left(\gamma-\kappa_{3}\right)^{\beta}}{\Gamma(\alpha+1) \Gamma(\beta+1)} \digamma\left(\kappa_{1}, \kappa_{3}\right)+\frac{\left(\varkappa-\kappa_{1}\right)^{\alpha}\left(\kappa_{4}-\gamma\right)^{\beta}}{\Gamma(\alpha+1) \Gamma(\beta+1)} \digamma\left(\kappa_{1}, \kappa_{4}\right)
$$




$$
\begin{aligned}
& +\frac{\left(\kappa_{2}-\varkappa\right)^{\alpha}\left(\gamma-\kappa_{3}\right)^{\beta}}{\Gamma(\alpha+1) \Gamma(\beta+1)} \digamma\left(\kappa_{2}, \kappa_{3}\right)+\frac{\left(\kappa_{2}-\varkappa\right)^{\alpha}\left(\kappa_{4}-\gamma\right)^{\beta}}{\Gamma(\alpha+1) \Gamma(\beta+1)} \digamma\left(\kappa_{2}, \kappa_{4}\right) \\
& -\frac{\left(\gamma-\kappa_{3}\right)^{\beta}}{\Gamma(\beta+1)}\left[\mathbf{J}_{\kappa_{1}+}^{\alpha} \digamma\left(\varkappa, \kappa_{3}\right)+\mathbf{J}_{\kappa_{2}-}^{\alpha} \digamma\left(\varkappa, \kappa_{3}\right)\right] \\
& -\frac{\left(\kappa_{4}-\gamma\right)^{\beta}}{\Gamma(\beta+1)}\left[\mathbf{J}_{\kappa_{1}+}^{\alpha} \digamma\left(\varkappa, \kappa_{4}\right)+\mathbf{J}_{\kappa_{2}-}^{\alpha} \digamma\left(\varkappa, \kappa_{4}\right)\right] \\
& -\frac{\left(\varkappa-\kappa_{1}\right)^{\alpha}}{\Gamma(\alpha+1)}\left[\mathbf{J}_{\kappa_{3}+}^{\beta} \digamma\left(\kappa_{1}, \gamma\right)+\mathbf{J}_{\kappa_{4}-}^{\beta} \digamma\left(\kappa_{1}, \gamma\right)\right] \\
& -\frac{\left(\kappa_{2}-\varkappa\right)^{\alpha}}{\Gamma(\alpha+1)}\left[\mathbf{J}_{\kappa_{3}+}^{\beta} \digamma\left(\kappa_{2}, \gamma\right)+\mathbf{J}_{\kappa_{4}-}^{\beta} \digamma\left(\kappa_{2}, \gamma\right)\right] \\
& +\mathbf{J}_{\kappa_{1}+, \kappa_{3}+}^{\alpha, \beta} \digamma(\varkappa, \gamma)+\mathbf{J}_{\kappa_{1}+, \kappa_{4}-}^{\alpha, \beta} \digamma(\varkappa, \gamma)+\mathbf{J}_{\kappa_{2}-, \kappa_{3}+}^{\alpha, \beta} \digamma(\varkappa, \gamma)+\mathbf{J}_{\kappa_{2}-, \kappa_{4}-}^{\alpha, \beta} \digamma(\varkappa, \gamma) \mid \\
& \leq \frac{1}{\Gamma(\alpha+1) \Gamma(\beta+1)} \\
& \times\left[\left(\varkappa-\kappa_{1}\right)^{\alpha}\left(\gamma-\kappa_{3}\right)^{\beta} \bigvee_{\kappa_{1}}^{\varkappa} \bigvee_{\kappa_{3}}^{\gamma}(\digamma)+\left(\varkappa-\kappa_{1}\right)^{\alpha}\left(\kappa_{4}-\gamma\right)^{\beta} \bigvee_{\kappa_{1}}^{\varkappa} \bigvee_{\gamma}^{\kappa_{4}}(\digamma)\right. \\
& \left.+\left(\kappa_{2}-\varkappa\right)^{\alpha}\left(\gamma-\kappa_{3}\right)^{\beta} \bigvee_{\varkappa}^{\kappa_{2}} \bigvee_{\kappa_{3}}^{\gamma}(\digamma)+\left(\kappa_{2}-\varkappa\right)^{\alpha}\left(\kappa_{4}-\gamma\right)^{\beta} \bigvee_{\varkappa}^{\kappa_{2}} \bigvee_{\gamma}^{\kappa_{4}}(\digamma)\right] \\
& \leq \frac{1}{\Gamma(\alpha+1) \Gamma(\beta+1)}\left[\frac{\kappa_{2}-\kappa_{1}}{2}+\left|\varkappa-\frac{\kappa_{1}+\kappa_{2}}{2}\right|\right]^{\alpha} \\
& \times\left[\frac{\kappa_{4}-\kappa_{3}}{2}+\left|\gamma-\frac{\kappa_{3}+\kappa_{4}}{2}\right|\right] \bigvee_{\kappa_{1}}^{\beta} \bigvee_{\kappa_{2}}^{\kappa_{2}}(\digamma) \text {. }
\end{aligned}
$$

Corollary 2. If we take $\varkappa=\frac{\kappa_{1}+\kappa_{2}}{2}$ and $\gamma=\frac{\kappa_{3}+\kappa_{4}}{2}$ in Corollary 1, then we have

$$
\begin{aligned}
& \mid \frac{\digamma\left(\kappa_{2}, \kappa_{4}\right)+\digamma\left(\kappa_{2}, \kappa_{3}\right)+\digamma\left(\kappa_{1}, \kappa_{4}\right)+\digamma\left(\kappa_{1}, \kappa_{3}\right)}{4} \\
& -\frac{2^{\alpha-1} \Gamma(\alpha+1)}{\left(\kappa_{2}-\kappa_{1}\right)^{\alpha}}\left[\mathbf{J}_{\kappa_{1}+}^{\alpha} \digamma\left(\varkappa, \kappa_{3}\right)+\mathbf{J}_{\kappa_{2}-}^{\alpha} \digamma\left(\varkappa, \kappa_{3}\right)\right] \\
& -\frac{2^{\alpha-1} \Gamma(\alpha+1)}{\left(\kappa_{2}-\kappa_{1}\right)^{\alpha}}\left[\mathbf{J}_{\kappa_{1}+}^{\alpha} \digamma\left(\varkappa, \kappa_{4}\right)+\mathbf{J}_{\kappa_{2}-}^{\alpha} \digamma\left(\varkappa, \kappa_{4}\right)\right] \\
& -\frac{2^{\beta-1} \Gamma(\beta+1)}{\left(\kappa_{4}-\kappa_{3}\right)^{\beta}}\left[\mathbf{J}_{\kappa_{3}+}^{\beta} \digamma\left(\kappa_{1}, \gamma\right)+\mathbf{J}_{\kappa_{4}-}^{\beta} \digamma\left(\kappa_{1}, \gamma\right)\right] \\
& -\frac{2^{\beta-1} \Gamma(\beta+1)}{\left(\kappa_{4}-\kappa_{3}\right)^{\beta}}\left[\mathbf{J}_{\kappa_{3}+}^{\beta} \digamma\left(\kappa_{2}, \gamma\right)+\mathbf{J}_{\kappa_{4}-}^{\beta} \digamma\left(\kappa_{2}, \gamma\right)\right] \\
& \frac{2^{\alpha+\beta-2} \Gamma(\alpha+1) \Gamma(\beta+1)}{\left(\kappa_{2}-\kappa_{1}\right)^{\alpha}\left(\kappa_{4}-\kappa_{3}\right)^{\beta}}\left[\mathbf{J}_{\kappa_{1}+, \kappa_{3}+}^{\alpha, \beta} \digamma(\varkappa, \gamma)+\mathbf{J}_{\kappa_{1}+, \kappa_{4}-}^{\alpha, \beta} \digamma(\varkappa, \gamma)\right. \\
& \left.+\mathbf{J}_{\kappa_{2}-, \kappa_{3}+}^{\alpha, \beta} \digamma(\varkappa, \gamma)+\mathbf{J}_{\kappa_{2}-, \kappa_{4}-}^{\alpha, \beta} \digamma(\varkappa, \gamma)\right] \mid \\
& \leq \frac{1}{4} \bigvee_{\kappa_{1}}^{\kappa_{2}} \bigvee_{\kappa_{2}}^{\kappa_{4}}(\digamma)
\end{aligned}
$$


Remark 1. If we assign $\alpha=\beta=1$ in Corollary 2, then we have

$$
\begin{aligned}
& \mid \frac{\digamma\left(\kappa_{2}, \kappa_{4}\right)+\digamma\left(\kappa_{2}, \kappa_{3}\right)+\digamma\left(\kappa_{1}, \kappa_{4}\right)+\digamma\left(\kappa_{1}, \kappa_{3}\right)}{4}\left[\int_{\kappa_{1}}^{\kappa_{2}} \digamma\left(\varkappa, \kappa_{4}\right) d \varkappa+\int_{\kappa_{1}}^{\kappa_{2}} \digamma\left(\varkappa, \kappa_{3}\right) d \varkappa\right] \\
& -\frac{1}{2\left(\kappa_{2}-\kappa_{1}\right)}\left[\int_{\kappa_{3}}^{\kappa_{4}} \digamma\left(\kappa_{2}, \gamma\right) d \gamma+\int_{\kappa_{3}}^{\kappa_{4}} \digamma\left(\kappa_{1}, \gamma\right) d \gamma\right] \\
& \quad-\frac{1}{2\left(\kappa_{4}-\kappa_{3}\right)} \int_{\kappa_{1}}^{\kappa_{2}} \int_{\kappa_{3}}^{\kappa_{4}} \digamma(\varkappa, \gamma) d \gamma d \varkappa \mid \\
& \leq \frac{1}{4} \bigvee_{\kappa_{1}}^{\kappa_{2} \aleph_{\kappa_{3}} \kappa_{4}(\digamma) .}
\end{aligned}
$$

which is given by Budak and Sarikaya in [43].

Theorem 3. If $\digamma: \Delta \rightarrow \mathbb{R}$ be a mapping of bounded variation on $\Delta$, then for $\alpha, \beta>0$, we have the following inequality

$$
\begin{aligned}
& \mid \frac{\digamma\left(\kappa_{2}, \kappa_{4}\right)+\digamma\left(\kappa_{2}, \kappa_{3}\right)+\digamma\left(\kappa_{1}, \kappa_{4}\right)+\digamma\left(\kappa_{1}, \kappa_{3}\right)}{4} \\
& \quad-\frac{1}{4 M_{\rho}^{\alpha}\left(\kappa_{1}, \kappa_{2}\right)}\left[\mathcal{J}_{\kappa_{2}-; \rho}^{\alpha} \digamma\left(\kappa_{1}, \kappa_{4}\right)+\mathcal{J}_{\kappa_{2}-; \rho}^{\alpha} \digamma\left(\kappa_{1}, \kappa_{3}\right)+\mathcal{J}_{\kappa_{1}+; \rho}^{\alpha} \digamma\left(\kappa_{2}, \kappa_{4}\right)+\mathcal{J}_{\kappa_{1}+; \rho}^{\alpha} \digamma\left(\kappa_{2}, \kappa_{3}\right)\right] \\
& \quad-\frac{1}{4 N_{\omega}^{\beta}\left(\kappa_{3}, \kappa_{4}\right)}\left[\mathcal{J}_{\kappa_{4}-; \omega}^{\beta} \digamma\left(\kappa_{2}, \kappa_{3}\right)+\mathcal{J}_{\kappa_{3}+; \omega}^{\beta} \digamma\left(\kappa_{2}, \kappa_{4}\right)+\mathcal{J}_{\kappa_{4}-; \omega}^{\beta} \digamma\left(\kappa_{1}, \kappa_{3}\right)+\mathcal{J}_{\kappa_{3}+; \omega}^{\beta} \digamma\left(\kappa_{1}, \kappa_{4}\right)\right] \\
& +\frac{1}{4 M_{\rho}^{\alpha}\left(\kappa_{1}, \kappa_{2}\right) N_{\omega}^{\beta}\left(\kappa_{3}, \kappa_{4}\right)}\left[\mathcal{J}_{\kappa_{2}-, \kappa_{4}-; \rho, \omega}^{\alpha, \beta} \digamma\left(\kappa_{1}, \kappa_{3}\right)+\mathcal{J}_{\kappa_{2}-, \kappa_{3}+; \rho, \omega}^{\alpha, \beta} \digamma\left(\kappa_{1}, \kappa_{4}\right)\right. \\
& \left.\quad+\mathcal{J}_{\kappa_{1}+, \kappa_{4}-; \rho, \omega}^{\alpha, \beta}\left(\kappa_{2}, \kappa_{3}\right)+\mathcal{J}_{\kappa_{1}+, \kappa_{3}+; \rho, \omega}^{\alpha, \beta} \digamma\left(\kappa_{2}, \kappa_{4}\right)\right] \mid \\
& \leq \quad \frac{1}{4} \bigvee_{\kappa_{1}}^{\kappa_{2} \bigvee_{\kappa_{3}} \aleph_{4}(\digamma) .}
\end{aligned}
$$

Proof. By using Lemma 2, we have

$$
\begin{aligned}
& \mid \frac{\digamma\left(\kappa_{2}, \kappa_{4}\right)+\digamma\left(\kappa_{2}, \kappa_{3}\right)+\digamma\left(\kappa_{1}, \kappa_{4}\right)+\digamma\left(\kappa_{1}, \kappa_{3}\right)}{4} \\
& -\frac{1}{4 M_{\rho}^{\alpha}\left(\kappa_{1}, \kappa_{2}\right)}\left[\mathcal{J}_{\kappa_{2}-; \rho}^{\alpha} \digamma\left(\kappa_{1}, \kappa_{4}\right)+\mathcal{J}_{\kappa_{2}-; \rho}^{\alpha} \digamma\left(\kappa_{1}, \kappa_{3}\right)\right. \\
& \left.+\mathcal{J}_{\kappa_{1}+; \rho}^{\alpha} \digamma\left(\kappa_{2}, \kappa_{4}\right)+\mathcal{J}_{\kappa_{1}+; \rho}^{\alpha} \digamma\left(\kappa_{2}, \kappa_{3}\right)\right] \\
& -\frac{1}{4 N_{\omega}^{\beta}\left(\kappa_{3}, \kappa_{4}\right)}\left[\mathcal{J}_{\kappa_{4}-; \omega}^{\beta} \digamma\left(\kappa_{2}, \kappa_{3}\right)+\mathcal{J}_{\kappa_{3}+; \omega}^{\beta} \digamma\left(\kappa_{2}, \kappa_{4}\right)\right. \\
& \left.+\mathcal{J}_{\kappa_{4}-; \omega}^{\beta} \digamma\left(\kappa_{1}, \kappa_{3}\right)+\mathcal{J}_{\kappa_{3}+; \omega}^{\beta} \digamma\left(\kappa_{1}, \kappa_{4}\right)\right]
\end{aligned}
$$




$$
\begin{aligned}
& +\frac{1}{4 M_{\rho}^{\alpha}\left(\kappa_{1}, \kappa_{2}\right) N_{\omega}^{\beta}\left(\kappa_{3}, \kappa_{4}\right)}\left[\mathcal{J}_{\kappa_{2}-, \kappa_{4}-; \rho, \omega}^{\alpha, \beta} \digamma\left(\kappa_{1}, \kappa_{3}\right)+\mathcal{J}_{\kappa_{2}-, \kappa_{3}+; \rho, \omega}^{\alpha, \beta} \digamma\left(\kappa_{1}, \kappa_{4}\right)\right. \\
& \left.+\mathcal{J}_{\kappa_{1}+, \kappa_{4}-; \rho, \omega}^{\alpha, \beta} \digamma\left(\kappa_{2}, \kappa_{3}\right)+\mathcal{J}_{\kappa_{1}+, \kappa_{3}+; \rho, \omega}^{\alpha, \beta} \digamma\left(\kappa_{2}, \kappa_{4}\right)\right] \mid \\
& =\frac{1}{4 \Gamma(\alpha+1) \Gamma(\beta+1)} \frac{1}{M_{\rho}^{\alpha}\left(\kappa_{1}, \kappa_{2}\right) N_{\omega}^{\beta}\left(\kappa_{3}, \kappa_{4}\right)} \\
& \left.\times \mid \int_{\kappa_{1}}^{\kappa_{2}} \int_{\kappa_{3}}^{\kappa_{2}}\left[\left(\rho\left(\kappa_{2}\right)-\rho(\tau)\right)^{\alpha}-\rho(\tau)-\rho\left(\kappa_{1}\right)\right)^{\alpha}\right] \\
& \times\left[\left(\omega\left(\kappa_{4}\right)-\omega(\xi)\right)^{\beta}-\left(\omega(\xi)-\omega\left(\kappa_{3}\right)\right)^{\beta}\right] d_{\tau} d_{\xi} \digamma(\tau, \xi) \mid \\
& \leq \frac{1}{4 \Gamma(\alpha+1) \Gamma(\beta+1)} \frac{1}{M_{\rho}^{\alpha}\left(\kappa_{1}, \kappa_{2}\right) N_{\omega}^{\beta}\left(\kappa_{3}, \kappa_{4}\right)} \\
& \left.\times \sup _{(\tau, \gamma) \in \Delta} \mid\left(\rho\left(\kappa_{2}\right)-\rho(\tau)\right)^{\alpha}-\rho(\tau)-\rho\left(\kappa_{1}\right)\right)^{\alpha} \mid \\
& \times\left|\left(\omega\left(\kappa_{4}\right)-\omega(\xi)\right)^{\beta}-\left(\omega(\xi)-\omega\left(\kappa_{3}\right)\right)^{\beta}\right| \bigvee_{\kappa_{1}}^{\kappa_{2}} \bigvee_{\kappa_{3}}^{\kappa_{4}}(\digamma) \\
& =\frac{1}{4 \Gamma(\alpha+1) \Gamma(\beta+1)} \frac{\left(\rho\left(\kappa_{2}\right)-\rho(\tau)\right)^{\alpha}\left(\omega\left(\kappa_{4}\right)-\omega(\xi)\right)^{\beta}}{M_{\rho}^{\alpha}\left(\kappa_{1}, \kappa_{2}\right) N_{\omega}^{\beta}\left(\kappa_{3}, \kappa_{4}\right)} \bigvee_{\kappa_{1}}^{\kappa_{\kappa_{3}}} \bigvee_{\kappa_{3}}(\digamma) \\
& =\frac{1}{4} \bigvee_{\kappa_{1}}^{\kappa_{2}} \bigvee_{\kappa_{3}}^{\kappa_{4}}(\digamma) \text {. }
\end{aligned}
$$

This completes the proof.

Corollary 3. If we take $\rho(\tau)=\tau, \tau \in\left[\kappa_{1}, \kappa_{2}\right]$ and $\omega(\xi)=\xi, \xi \in\left[\kappa_{3}, \kappa_{4}\right]$ in Theorem 3 , then we have

$$
\begin{aligned}
& \mid \frac{\digamma\left(\kappa_{2}, \kappa_{4}\right)+\digamma\left(\kappa_{2}, \kappa_{3}\right)+\digamma\left(\kappa_{1}, \kappa_{4}\right)+\digamma\left(\kappa_{1}, \kappa_{3}\right)}{4} \\
& \quad-\frac{\Gamma(\alpha+1)}{4\left(\kappa_{2}-\kappa_{1}\right)^{\alpha}}\left[\mathbf{J}_{\kappa_{2}-}^{\alpha} \digamma\left(\kappa_{1}, \kappa_{4}\right)+\mathbf{J}_{\kappa_{2}-}^{\alpha} \digamma\left(\kappa_{1}, \kappa_{3}\right)+\mathbf{J}_{\kappa_{1}+}^{\alpha} \digamma\left(\kappa_{2}, \kappa_{4}\right)+\mathbf{J}_{\kappa_{1}+}^{\alpha} \digamma\left(\kappa_{2}, \kappa_{3}\right)\right] \\
& \quad-\frac{\Gamma(\beta+1)}{4\left(\kappa_{4}-\kappa_{3}\right)^{\beta}}\left[\mathbf{J}_{\kappa_{4}-}^{\beta} \digamma\left(\kappa_{2}, \kappa_{3}\right)+\mathbf{J}_{\kappa_{3}+}^{\beta} \digamma\left(\kappa_{2}, \kappa_{4}\right)+\mathbf{J}_{\kappa_{4}-}^{\beta} \digamma\left(\kappa_{1}, \kappa_{3}\right)+\mathbf{J}_{\kappa_{3}+}^{\beta} \digamma\left(\kappa_{1}, \kappa_{4}\right)\right] \\
& \quad+\frac{\Gamma(\alpha+1) \Gamma(\beta+1)}{4\left(\kappa_{2}-\kappa_{1}\right)^{\alpha}\left(\kappa_{4}-\kappa_{3}\right)^{\beta}}\left[\mathbf{J}_{\kappa_{2}-, \kappa_{4}-}^{\alpha, \beta} \digamma\left(\kappa_{1}, \kappa_{3}\right)+\mathbf{J}_{\kappa_{2}-, \kappa_{3}+}^{\alpha, \beta} \digamma\left(\kappa_{1}, \kappa_{4}\right)\right. \\
& \left.\quad+\mathbf{J}_{\kappa_{1}+, \kappa_{4}-}^{\alpha, \beta}\left(\kappa_{2}, \kappa_{3}\right)+\mathbf{J}_{\kappa_{1}+, \kappa_{3}+}^{\alpha, \beta} \digamma\left(\kappa_{2}, \kappa_{4}\right)\right] \mid \\
& \leq \quad \frac{1}{4} \bigvee_{\kappa_{1} \kappa_{\kappa_{3}} \aleph_{2} \kappa_{4}}^{\kappa_{1}}(\digamma) .
\end{aligned}
$$

Remark 2. If we assign $\alpha=\beta=1$ in Corollary 3 , then we have

$$
\begin{aligned}
& \mid \frac{\digamma\left(\kappa_{2}, \kappa_{4}\right)+\digamma\left(\kappa_{2}, \kappa_{3}\right)+\digamma\left(\kappa_{1}, \kappa_{4}\right)+\digamma\left(\kappa_{1}, \kappa_{3}\right)}{4} \\
& -\frac{1}{2\left(\kappa_{2}-\kappa_{1}\right)}\left[\int_{\kappa_{1}}^{\kappa_{2}} \digamma\left(\varkappa, \kappa_{4}\right) d \varkappa+\int_{\kappa_{1}}^{\kappa_{2}} \digamma\left(\varkappa, \kappa_{3}\right) d \varkappa\right]
\end{aligned}
$$




$$
\begin{aligned}
& -\frac{1}{2\left(\kappa_{4}-\kappa_{3}\right)}\left[\int_{\kappa_{3}}^{\kappa_{4}} \digamma\left(\kappa_{2}, \gamma\right) d \gamma+\int_{\kappa_{3}}^{\kappa_{4}} \digamma\left(\kappa_{1}, \gamma\right) d \gamma\right] \\
& +\frac{1}{\left(\kappa_{2}-\kappa_{1}\right)\left(\kappa_{4}-\kappa_{3}\right)} \int_{\kappa_{1}}^{\kappa_{2}} \int_{\kappa_{3}}^{\kappa_{4}} \digamma(\varkappa, \gamma) d \gamma d \varkappa \mid \\
& \leq \frac{1}{4} \bigvee_{\kappa_{1}}^{\kappa_{2}} \bigvee_{\kappa_{3}}^{\kappa_{4}}(\digamma) .
\end{aligned}
$$

which is given by Budak and Sarikaya in [43].

Corollary 4. If we take $\rho(\tau)=\ln \tau, \tau \in\left[\kappa_{1}, \kappa_{2}\right]$ and $\omega(\xi)=\ln \xi, \xi \in\left[\kappa_{3}, \kappa_{4}\right]$ in Theorem 3, then we have the following trapezoid-type inequality for Hadamard fractional integrals

$$
\begin{aligned}
& \mid \frac{\digamma\left(\kappa_{2}, \kappa_{4}\right)+\digamma\left(\kappa_{2}, \kappa_{3}\right)+\digamma\left(\kappa_{1}, \kappa_{4}\right)+\digamma\left(\kappa_{1}, \kappa_{3}\right)}{4} \\
& -\frac{1}{4 M_{\mathrm{ln}}^{\alpha}\left(\kappa_{1}, \kappa_{2}\right)}\left[I_{\kappa_{2}-}^{\alpha} \digamma\left(\kappa_{1}, \kappa_{4}\right)+I_{\kappa_{2}-}^{\alpha} \digamma\left(\kappa_{1}, \kappa_{3}\right)+I_{\kappa_{1}+}^{\alpha} \digamma\left(\kappa_{2}, \kappa_{4}\right)+I_{\kappa_{1}+}^{\alpha} \digamma\left(\kappa_{2}, \kappa_{3}\right)\right] \\
& -\frac{1}{4 N_{\ln }^{\beta}\left(\kappa_{3}, \kappa_{4}\right)}\left[I_{\kappa_{4}-}^{\beta} \digamma\left(\kappa_{2}, \kappa_{3}\right)+I_{\kappa_{3}+}^{\beta} \digamma\left(\kappa_{2}, \kappa_{4}\right)+I_{\kappa_{4}-}^{\beta} \digamma\left(\kappa_{1}, \kappa_{3}\right)+I_{\kappa_{3}+}^{\beta} \digamma\left(\kappa_{1}, \kappa_{4}\right)\right] \\
& +\frac{1}{4 M_{\ln }^{\alpha}\left(\kappa_{1}, \kappa_{2}\right) N_{\ln }^{\beta}\left(\kappa_{3}, \kappa_{4}\right)}\left[I_{\kappa_{2}-, \kappa_{4}-}^{\alpha, \beta} \digamma\left(\kappa_{1}, \kappa_{3}\right)+I_{\kappa_{2}-, \kappa_{3}+}^{\alpha, \beta} \digamma\left(\kappa_{1}, \kappa_{4}\right)\right. \\
& \left.+I_{\kappa_{1}+, \kappa_{4}-}^{\alpha, \beta} \digamma\left(\kappa_{2}, \kappa_{3}\right)+I_{\kappa_{1}+, \kappa_{3}+}^{\alpha, \beta} \digamma\left(\kappa_{2}, \kappa_{4}\right)\right] \mid \\
& \leq \frac{1}{4} \bigvee_{\kappa_{1}}^{\kappa_{2}} \bigvee_{\kappa_{3}}^{\kappa_{4}}(\digamma)
\end{aligned}
$$

\section{Ostrowski-Type Inequalities for Functions of Bounded Variations with} Two Variables

In this section, we prove some Ostrowski-type inequalities for generalized fractional integrals.

Theorem 4. If $\digamma: \Delta \rightarrow \mathbb{R}$ be a mapping of bounded variation on $\Delta$, then for $\alpha, \beta>0$, we have the following inequality

$$
\begin{aligned}
& \mid \digamma(\varkappa, \gamma)-\frac{\Gamma(\alpha+1)}{M_{\rho}^{\alpha}\left(\kappa_{1}, \kappa_{2} ; \varkappa\right)}\left[\mathcal{J}_{\varkappa-; \rho}^{\alpha} \digamma\left(\kappa_{1}, \gamma\right)+\mathcal{J}_{\varkappa+; \rho}^{\alpha} \digamma\left(\kappa_{2}, \gamma\right)\right] \\
& -\frac{\Gamma(\beta+1)}{N_{\omega}^{\beta}\left(\kappa_{3}, \kappa_{4} ; \gamma\right)}\left[\mathcal{J}_{\gamma-; \omega}^{\beta} \digamma\left(\varkappa, \kappa_{3}\right)+\mathcal{J}_{\gamma+\infty}^{\beta} \digamma\left(\varkappa, \kappa_{4}\right)\right] \\
& +\frac{\Gamma(\alpha+1) \Gamma(\beta+1)}{M_{\rho}^{\alpha}\left(\kappa_{1}, \kappa_{2} ; \varkappa\right) N_{\omega}^{\beta}\left(\kappa_{3}, \kappa_{4} ; \gamma\right)}\left[\mathcal{J}_{\varkappa-, \gamma-; \rho, \omega}^{\alpha, \beta} \digamma\left(\kappa_{1}, \kappa_{3}\right)+\mathcal{J}_{\varkappa-, \gamma+;, \omega}^{\alpha, \beta} \digamma\left(\kappa_{1}, \kappa_{4}\right)\right. \\
& \left.+\mathcal{J}_{\varkappa+, \gamma-; \rho, \omega}^{\alpha, \beta} \digamma\left(\kappa_{2}, \kappa_{3}\right)+\mathcal{J}_{\varkappa+, \gamma+; \rho, \omega}^{\alpha, \beta} \digamma\left(\kappa_{2}, \kappa_{4}\right)\right] \mid \\
& =\frac{1}{M_{\rho}^{\alpha}\left(\kappa_{1}, \kappa_{2} ; \varkappa\right) N_{\omega}^{\beta}\left(\kappa_{3}, \kappa_{4} ; \gamma\right)}
\end{aligned}
$$




$$
\begin{aligned}
& \times\left[\left(\rho(\varkappa)-\rho\left(\kappa_{1}\right)\right)^{\alpha}\left(\omega(\gamma)-\omega\left(\kappa_{3}\right)\right)^{\beta} \bigvee_{\kappa_{1}}^{\varkappa} \bigvee_{\kappa_{3}}^{\gamma}(\digamma)\right. \\
& +\left(\rho(\varkappa)-\rho\left(\kappa_{1}\right)\right)^{\alpha}\left(\omega\left(\kappa_{4}\right)-\omega(\gamma)\right)^{\beta} \bigvee_{\kappa_{1}}^{\varkappa} \bigvee_{\gamma}^{\kappa_{4}}(\digamma) \\
& +\left(\rho\left(\kappa_{2}\right)-\rho(\varkappa)\right)^{\alpha}\left(\omega(\gamma)-\omega\left(\kappa_{3}\right)\right)^{\beta} \bigvee_{\varkappa}^{\kappa_{2}} \bigvee_{\kappa_{3}}^{\gamma}(\digamma) \\
& \left.+\left(\rho\left(\kappa_{2}\right)-\rho(\varkappa)\right)^{\alpha}\left(\omega\left(\kappa_{4}\right)-\omega(\gamma)\right)^{\beta} \bigvee_{\varkappa}^{\kappa_{2}} \bigvee_{\gamma}^{\kappa_{4}}(\digamma)\right] \\
& \leq \frac{1}{M_{\rho}^{\alpha}\left(\kappa_{1}, \kappa_{2} ; \varkappa\right) N_{\omega}^{\beta}\left(\kappa_{3}, \kappa_{4} ; \gamma\right)} \\
& \times\left[\frac{1}{2}\left(\rho\left(\kappa_{2}\right)-\rho\left(\kappa_{1}\right)\right)+\left|\rho(\varkappa)-\frac{\rho\left(\kappa_{1}\right)+\rho\left(\kappa_{2}\right)}{2}\right|\right]^{\alpha} \\
& \times\left[\frac{1}{2}\left(\omega\left(\kappa_{4}\right)-\omega\left(\kappa_{3}\right)\right)+\left|\omega(\gamma)-\frac{\omega\left(\kappa_{3}\right)+\omega\left(\kappa_{4}\right)}{2}\right|\right] \bigvee_{\kappa_{1}}^{\beta} \bigvee_{\kappa_{2}}^{\kappa_{2}}(\digamma) \text {. }
\end{aligned}
$$

Proof. By taking the modulus in Lemma 3, we obtain

$$
\begin{aligned}
& \mid \digamma(\varkappa, \gamma)-\frac{\Gamma(\alpha+1)}{M_{\rho}^{\alpha}\left(\kappa_{1}, \kappa_{2} ; \varkappa\right)}\left[\mathcal{J}_{\varkappa-; \rho}^{\alpha} \digamma\left(\kappa_{1}, \gamma\right)+\mathcal{J}_{\varkappa+; \rho}^{\alpha} \digamma\left(\kappa_{2}, \gamma\right)\right] \\
& +\frac{\Gamma(\beta+1)}{N_{\omega}^{\beta}\left(\kappa_{3}, \kappa_{4} ; \gamma\right)}\left[\mathcal{J}_{\gamma-; \omega}^{\beta} \digamma\left(\varkappa, \kappa_{3}\right)+\mathcal{J}_{\gamma+; \omega}^{\beta} \digamma\left(\varkappa, \kappa_{4}\right)\right] \\
& +\frac{\Gamma(\alpha+1) \Gamma(\beta+1)}{M_{\rho}^{\alpha}\left(\kappa_{1}, \kappa_{2} ; \varkappa\right) N_{\omega}^{\beta}\left(\kappa_{3}, \kappa_{4} ; \gamma\right)}\left[\mathcal{J}_{\varkappa-, \gamma-; \rho, \omega}^{\alpha, \beta} \digamma\left(\kappa_{1}, \kappa_{3}\right)+\mathcal{J}_{\varkappa-, \gamma+; \rho, \omega}^{\alpha, \beta} \digamma\left(\kappa_{1}, \kappa_{4}\right)\right. \\
& \left.+\mathcal{J}_{\varkappa+, \gamma-; \rho, \omega}^{\alpha, \beta} \digamma\left(\kappa_{2}, \kappa_{3}\right)+\mathcal{J}_{\varkappa+, \gamma+; \rho, \omega}^{\alpha, \beta}\left(\kappa_{2}, \kappa_{4}\right)\right] \mid \\
& \quad M_{\rho}^{\alpha}\left(\kappa_{1}, \kappa_{2} ; \varkappa\right) N_{\omega}^{\beta}\left(\kappa_{3}, \kappa_{4} ; \gamma\right) \\
& +|| \int_{\kappa_{1}}^{\varkappa} \int_{\kappa_{3}}^{\gamma}\left(\rho(\tau)-\rho\left(\kappa_{1}\right)\right)^{\alpha}\left(\omega(\xi)-\omega\left(\kappa_{3}\right)\right)^{\beta} d_{\tau} d_{\xi} \digamma(\tau, \xi) \mid \\
& +\left|\int_{\kappa_{1}}^{\kappa_{\gamma}} \int_{\gamma}^{\kappa_{4}}\left(\rho(\tau)-\rho\left(\kappa_{1}\right)\right)^{\alpha}\left(\omega\left(\kappa_{4}\right)-\omega(\xi)\right)^{\beta} d_{\tau} d_{\xi} \digamma(\tau, \xi)\right| \\
& +\mid \int_{\varkappa}^{\kappa_{2}} \gamma \\
& +\left|\int_{\kappa_{3}}^{\gamma}\left(\rho\left(\kappa_{2}\right)-\rho(\tau)\right)^{\alpha}\left(\omega(\xi)-\omega\left(\kappa_{3}\right)\right)^{\beta} d_{\tau} d_{\xi} \digamma(\tau, \xi)\right|
\end{aligned}
$$

By Lemma 2, we obtain

$$
\left|\int_{\kappa_{1}}^{\varkappa} \int_{\kappa_{3}}^{\gamma}\left(\rho(\tau)-\rho\left(\kappa_{1}\right)\right)^{\alpha}\left(\omega(\xi)-\omega\left(\kappa_{3}\right)\right)^{\beta} d_{\tau} d_{\xi} \digamma(\tau, \xi)\right|
$$




$$
\begin{array}{r}
\leq\left(\rho(\varkappa)-\rho\left(\kappa_{1}\right)\right)^{\alpha}\left(\omega(\gamma)-\omega\left(\kappa_{3}\right)\right)^{\beta} \bigvee_{\kappa_{1}}^{\varkappa} \bigvee_{\kappa_{3}}^{\gamma}(\digamma), \\
\left|\int_{\kappa_{1}}^{\varkappa} \int_{\gamma}^{\kappa_{4}}\left(\rho(\tau)-\rho\left(\kappa_{1}\right)\right)^{\alpha}\left(\omega\left(\kappa_{4}\right)-\omega(\xi)\right)^{\beta} d_{\tau} d_{\xi} \digamma(\tau, \xi)\right| \\
\leq\left(\rho(\varkappa)-\rho\left(\kappa_{1}\right)\right)^{\alpha}\left(\omega\left(\kappa_{4}\right)-\omega(\gamma)\right)^{\beta} \bigvee_{\kappa_{1}}^{\varkappa} \bigvee_{\gamma}^{\kappa_{4}}(\digamma), \\
\leq \int_{\varkappa}^{\kappa_{2}} \int_{\kappa_{3}}^{\gamma}\left(\rho\left(\kappa_{2}\right)-\rho(\tau)\right)^{\alpha}\left(\omega(\xi)-\omega\left(\kappa_{3}\right)\right)^{\beta} d_{\tau} d_{\xi} \digamma(\tau, \xi) \mid \\
\leq\left(\rho\left(\kappa_{2}\right)-\rho(\varkappa)\right)^{\alpha}\left(\omega(\gamma)-\omega\left(\kappa_{3}\right)\right)^{\beta} \bigvee_{\varkappa}^{\kappa_{2}} \bigvee_{\kappa_{3}}^{\gamma}(\digamma)
\end{array}
$$

and

$$
\begin{aligned}
& \mid \int_{\varkappa}^{\kappa_{2}} \int_{\gamma}^{\kappa_{4}}\left(\rho\left(\kappa_{2}\right)-\rho(\tau)\right)^{\alpha}\left(\omega\left(\kappa_{4}\right)-\omega(\xi)\right)^{\beta} d_{\tau} d_{\xi} \digamma(\tau, \xi) \mid \\
& \leq\left(\rho\left(\kappa_{2}\right)-\rho(\varkappa)\right)^{\alpha}\left(\omega\left(\kappa_{4}\right)-\omega(\gamma)\right)^{\beta} \bigvee_{\varkappa}^{\kappa_{2}} \bigvee_{\gamma}^{\kappa_{4}}(\digamma) .
\end{aligned}
$$

It follows that

$$
\begin{aligned}
& \mid \digamma(\varkappa, \gamma)-\frac{\Gamma(\alpha+1)}{M_{\rho}^{\alpha}\left(\kappa_{1}, \kappa_{2} ; \varkappa\right)}\left[\mathcal{J}_{\varkappa-; \rho}^{\alpha} \digamma\left(\kappa_{1}, \gamma\right)+\mathcal{J}_{\varkappa+; \rho}^{\alpha} \digamma\left(\kappa_{2}, \gamma\right)\right] \\
& -\frac{\Gamma(\beta+1)}{N_{\omega}^{\beta}\left(\kappa_{3}, \kappa_{4} ; \gamma\right)}\left[\mathcal{J}_{\gamma-; \omega}^{\beta} \digamma\left(\varkappa, \kappa_{3}\right)+\mathcal{J}_{\gamma+; \omega}^{\beta} \digamma\left(\varkappa, \kappa_{4}\right)\right] \\
& +\frac{\Gamma(\alpha+1) \Gamma(\beta+1)}{M_{\rho}^{\alpha}\left(\kappa_{1}, \kappa_{2} ; \varkappa\right) N_{\omega}^{\beta}\left(\kappa_{3}, \kappa_{4} ; \gamma\right)}\left[\mathcal{J}_{\varkappa-, \gamma-; \rho, \omega}^{\alpha, \beta} \digamma\left(\kappa_{1}, \kappa_{3}\right)+\mathcal{J}_{\varkappa-, \gamma+;, \omega}^{\alpha, \beta} \digamma\left(\kappa_{1}, \kappa_{4}\right)\right. \\
& \left.+\mathcal{J}_{\varkappa+, \beta}^{\alpha, \gamma-; \rho, \omega} \digamma\left(\kappa_{2}, \kappa_{3}\right)+\mathcal{J}_{\varkappa+, \gamma+; \rho, \omega}^{\alpha, \beta} \digamma\left(\kappa_{2}, \kappa_{4}\right)\right] \mid \\
& =\frac{1}{M_{\rho}^{\alpha}\left(\kappa_{1}, \kappa_{2} ; \varkappa\right) N_{\omega}^{\beta}\left(\kappa_{3}, \kappa_{4} ; \gamma\right)} \\
& +\left[\left(\rho(\varkappa)-\rho\left(\kappa_{1}\right)\right)^{\alpha}\left(\omega(\gamma)-\omega\left(\kappa_{3}\right)\right)^{\beta} \bigvee_{\kappa_{1}}^{\varkappa} \bigvee_{\kappa_{3}}^{\gamma}(\digamma)\right. \\
& +\left(\rho(\varkappa)-\rho\left(\kappa_{1}\right)\right)^{\alpha}\left(\omega\left(\kappa_{4}\right)-\omega(\gamma)\right)^{\beta} \bigvee_{\kappa_{1}}^{\varkappa} \bigvee_{\gamma}^{\kappa_{4}}(\digamma) \\
& +\left(\rho\left(\kappa_{2}\right)-\rho(\varkappa)\right)^{\alpha}\left(\omega(\gamma)-\omega\left(\kappa_{3}\right)\right)^{\beta} \bigvee_{\varkappa}^{\kappa_{2}} \bigvee_{\kappa_{3}}^{\gamma}(\digamma) \\
& \left.+\left(\rho\left(\kappa_{2}\right)-\rho(\varkappa)\right)^{\alpha}\left(\omega\left(\kappa_{4}\right)-\omega(\gamma)\right)^{\beta} \bigvee_{\varkappa}^{\kappa_{2}} \bigvee_{\gamma}^{\kappa_{4}}(\digamma)\right]
\end{aligned}
$$

which completes the proof of first inequality in Equation (28). The proof of the second inequality is obvious from the proof of Theorem 2. 
Remark 3. If we take $\rho(\tau)=\tau, \tau \in\left[\kappa_{1}, \kappa_{2}\right]$ and $\omega(\xi)=\xi, \xi \in\left[\kappa_{3}, \kappa_{4}\right]$ in Theorem 4, then we have the following Ostrowski-type inequalities for Riemann-Liouville fractional integrals

$$
\begin{aligned}
& \mid \digamma(\varkappa, \gamma)-\frac{\Gamma(\alpha+1)}{\left(\varkappa-\kappa_{1}\right)^{\alpha}+\left(\kappa_{2}-\varkappa\right)^{\alpha}}\left[\mathcal{J}_{\varkappa-; \rho}^{\alpha} \digamma\left(\kappa_{1}, \gamma\right)+\mathcal{J}_{\varkappa+; \rho}^{\alpha} \digamma\left(\kappa_{2}, \gamma\right)\right] \\
& -\frac{\Gamma(\beta+1)}{\left(\gamma-\kappa_{3}\right)^{\beta}+\left(\kappa_{4}-\gamma\right)^{\beta}}\left[\mathcal{J}_{\gamma-; \omega}^{\beta} \digamma\left(\varkappa, \kappa_{3}\right)+\mathcal{J}_{\gamma+\omega}^{\beta} \digamma\left(\varkappa, \kappa_{4}\right)\right] \\
& +\frac{\Gamma(\alpha+1) \Gamma(\beta+1)}{\left[\left(\varkappa-\kappa_{1}\right)^{\alpha}+\left(\kappa_{2}-\varkappa\right)^{\alpha}\right]\left[\left(\gamma-\kappa_{3}\right)^{\beta}+\left(\kappa_{4}-\gamma\right)^{\beta}\right]}\left[\mathcal{J}_{\varkappa-, \gamma-; \rho, \omega}^{\alpha, \beta} \digamma\left(\kappa_{1}, \kappa_{3}\right)\right. \\
& \left.+\mathcal{J}_{\varkappa-, \gamma+; \rho, \omega}^{\alpha, \beta} \digamma\left(\kappa_{1}, \kappa_{4}\right)+\mathcal{J}_{\varkappa+, \gamma-; \rho, \omega}^{\alpha, \beta} \digamma\left(\kappa_{2}, \kappa_{3}\right)+\mathcal{J}_{\varkappa+, \gamma+; \rho, \omega}^{\alpha, \beta} \digamma\left(\kappa_{2}, \kappa_{4}\right)\right] \mid \\
& =\frac{1}{\left[\left(\varkappa-\kappa_{1}\right)^{\alpha}+\left(\kappa_{2}-\varkappa\right)^{\alpha}\right]\left[\left(\gamma-\kappa_{3}\right)^{\beta}+\left(\kappa_{4}-\gamma\right)^{\beta}\right]} \\
& \times\left[\left(\varkappa-\kappa_{1}\right)^{\alpha}\left(\gamma-\kappa_{3}\right)^{\beta} \bigvee_{\kappa_{1}}^{\varkappa} \bigvee_{\kappa_{3}}^{\gamma}(\digamma)+\left(\varkappa-\kappa_{1}\right)^{\alpha}\left(\kappa_{4}-\gamma\right)^{\beta} \bigvee_{\kappa_{1}}^{\varkappa} \bigvee_{\gamma}^{\kappa_{4}}(\digamma)\right. \\
& \left.+\left(\kappa_{2}-\varkappa\right)^{\alpha}\left(\gamma-\kappa_{3}\right)^{\beta} \bigvee_{\varkappa}^{\kappa_{2}} \bigvee_{\kappa_{3}}^{\gamma}(\digamma)+\left(\kappa_{2}-\varkappa\right)^{\alpha}\left(\kappa_{4}-\gamma\right)^{\beta} \bigvee_{\varkappa}^{\kappa_{2}} \bigvee_{\gamma}^{\kappa_{4}}(\digamma)\right] \\
& \leq \frac{1}{\left[\left(\varkappa-\kappa_{1}\right)^{\alpha}+\left(\kappa_{2}-\varkappa\right)^{\alpha}\right]\left[\left(\gamma-\kappa_{3}\right)^{\beta}+\left(\kappa_{4}-\gamma\right)^{\beta}\right]} \\
& \times\left[\frac{\kappa_{2}-\kappa_{1}}{2}+\left|\varkappa-\frac{\kappa_{1}+\kappa_{2}}{2}\right|\right]^{\alpha}\left[\frac{\kappa_{4}-\kappa_{1}}{2}+\left|\gamma-\frac{\kappa_{3}+\kappa_{4}}{2}\right|\right]^{\beta} \bigvee_{\kappa_{1}}^{\kappa_{2}} \bigvee_{\kappa_{2}}^{\kappa_{4}}(\digamma) .
\end{aligned}
$$

\section{Conclusions}

In this paper, we present several trapezoid and Ostrowski-type inequalities for functions of bounded variation with two variables via generalized fractional integrals. It is also shown that several results are given by special cases of the main results. We deduce that the findings proved in this work are naturally universal, contribute to the theory of inequalities and have applications for determining the uniqueness of solutions in fractional boundary value problems. The findings of this study can be applied to symmetry. The results for the case of symmetric functions can be obtained by applying the concepts of symmetric convex functions, which will be studied in future work. It is an interesting and new problem and forthcoming researchers can use the techniques of this study to derive similar inequalities for different kinds of fractional integrals in their future works.

Author Contributions: T.S., H.B., H.K., M.A.A. and J.R. contributed equally to the writing of this paper. All authors have read and agreed to the published version of the manuscript.

Funding: This research was funded by King Mongkut's University of Technology North Bangkok. Contract no. KMUTNB-62-KNOW-26.

Institutional Review Board Statement: Not applicable.

Informed Consent Statement: Not applicable.

Data Availability Statement: Not applicable.

Acknowledgments: The authors would like to express their sincere thanks to the editor and the anonymous reviewers for their helpful comments and suggestions.

Conflicts of Interest: The authors declare no conflict of interest. 


\section{References}

1. Ostrowski, A. Über die Absolutabweichung einer differentiierbaren Funktion von ihrem Integralmittelwert. Comment. Math. Helv. 1938, 10, 226-227. [CrossRef]

2. Alomari, M.; Darus, M.; Dragomir, S.S.; Cerone, P. Ostrowski type inequalities for functions whose derivatives are $\xi$-convex in the second sense. Appl. Math. Lett. 2010, 23, 1071-1076. [CrossRef]

3. Kavurmaci, H.; Özdemir, M.E. New Ostrowski type inequalities for $m$-convex functions and applications. Hacet. J. Math. Stat. 2011, 40, 135-145.

4. Matłoka, M. Ostrowski type inequalities for functions whose derivatives are $h$-convex via fractional integrals. J. Sci. Res. Rep. 2014, 1633-1641. [CrossRef]

5. Özdemir, M.E.; Kavurmaci, H.; Set, E. Ostrowski's type inequalities for $(\alpha, m)$-convex function. Kyungpook Math. J. 2010, 50, 371-378. [CrossRef]

6. Tunç, M. Ostrowski-type inequalities via $h$-convex functions with applications to special means. J. Inequal. Appl. 2013, 2013, 326. [CrossRef]

7. Set, E. New inequalities of Ostrowski type for mappings whose derivatives are s-convex in the second sense via fractional integrals. Comput. Math. Appl. 2012, 63, 1147-1154. [CrossRef]

8. Farid, G.; Rehman, A.; Usman, M. Ostrowski type fractional integral inequalities for s-Godunova-Levin functions via $k$-fractional integrals. Proyecc. J. Math. 2017, 36, 753-767.

9. Sarikaya, M.Z.; Budak, H. Generalized Ostrowski type inequalities for local fractional integrals. Proc. Am. Math. Soc.2017, 145, 1527-1538. [CrossRef]

10. Agarwal, R.P.; Luo, M.-J.; Raina, R.K. On Ostrowski type inequalities. Fasc. Math. 2016, 56, 5-27. [CrossRef]

11. Basci, Y.; Baleanu, D. Ostrowski type inequalities involving $\psi$-Hilfer fractional integrals. Mathematics 2019, 7, 770. [CrossRef]

12. Budak, H.; Özçelik, K. Some generalized fractional trapezoid and Ostrowski type inequalities for functions with bounded partial derivatives. Math. Methods Appl. Sci. 2021, 1-21. [CrossRef]

13. Dragomir, S.S. Ostrowski and trapezoid type inequalities for the generalized $k$ - $\rho$-fractional integrals of functions with bounded variation. Commun. Adv. Math. Sci. 2017, 2, 309-330. [CrossRef]

14. Dragomir, S.S. Ostrowski and trapezoid type inequalities for Riemann-Liouville fractional integrals of absolutely continuous functions with bounded derivatives. Fract. Differ. Calc. 2020, 10, 307-320. [CrossRef]

15. Dragomir, S.S. On some trapezoid type inequalities for generalized Riemann-Liouville fractional integrals. RGMIA Res. Rep. Coll. 2017, 20, 12.

16. Dragomir, S.S. On some Ostrowski type inequalities for generalized Riemann-Liouville fractional integrals. RGMIA Res. Rep. Coll. 2017, 20, 13.

17. Dragomir, S.S. Ostrowski and trapezoid type inequalities for generalized Riemann-Liouville fractional integrals of absolutely continuous functions with bounded derivatives. RGMIA Res. Rep. Coll. 2017, 20, 19.

18. Dragomir, S.S. Further Ostrowski and trapezoid type inequalities for the generalized Riemann-Liouville fractional integrals of functions with bounded variation. RGMIA Res. Rep. Coll. 2017, 20, 20.

19. Farid, G.; Usman, M. Ostrowski type $k$-fractional integral inequalities for MT-convex and $h$-convex functions. Nonlinear Funct. Anal. Appl. 2017, 22, 627-639.

20. Gürbüz, M.; Taşdan, Y.; Set, E. Ostrowski type inequalities via the Katugampola fractional integrals. AIMS Math. 2020, 5, 42-53. [CrossRef]

21. Sarikaya, M.Z.; Filiz, H. Note on the Ostrowski type inequalities for fractional integrals. Vietnam J. Math. 2014, 42, 187-190. [CrossRef]

22. Sarikaya, M.Z.; Yildiz, M.K. Generalization and improvement of Ostrowski type inequalities. AIP Conf. Proc. 2018, $1991,020031$.

23. Sarikaya, M.Z.; Budak, H.; Yaldiz, H. Some new Ostrowski type inequalities for co-ordinated convex functions. Turkish J. Anal. Number Theory 2014, 2, 176-182. [CrossRef]

24. Latif, M.A.; Hussain, S.; Dragomir, S.S. New Ostrowski type inequalities for co-ordinated convex functions. TJMM 2012, 4, 125-136.

25. Latif, M.A.; Hussain, S. New inequalities of Ostrowski type for co-ordinated convex functions via fractional integrals. J. Fract. Calc. Appl. 2012, 2, 1-15.

26. Dragomir, S.S. On the Ostrowski's integral inequality for mappings with bounded variation and applications. Math. Inequal. Appl. 2001, 4, 59-66. [CrossRef]

27. Dragomir, S.S. On trapezoid quadrature formula and applications. Kragujev. J. Math. 2001, 23, 25-36.

28. Dragomir, S.S. On the midpoint quadrature formula for mappings with bounded variation and applications. Kragujev. J. Math. 2000, 22, 13-19.

29. Dragomir, S.S. On Simpson's quadrature formula for mappings of bounded variation and applications. Tamkang J. Math. 1999, 30, 53-58. [CrossRef]

30. Alomari, M.W. A generalization of Dragomir's generalization of Ostrowski integral inequality and applications in numerical integration. Ukr. Math. 2012, 64, 435-450.

31. Alomari, M.W. A Generalization of weighted companion of Ostrowski integral inequality for mappings of bounded variation. Int. J. Nonlinear Sci. Numer. Simul. 2020, 1, 667-673. [CrossRef] 
32. Barnett, N.S.; Dragomir, S.S.; Gomm, I. A companion for the Ostrowski and the generalized trapezoid inequalities. Math. Comput. Model. 2009, 50, 179-187. [CrossRef]

33. Budak, H.; Sarikaya, M.Z.; Qayyum, A. Improvement in companion of Ostrowski type inequalities for mappings whose first derivatives are of bounded variation and application. Filomat 2017, 31, 5305-5314. [CrossRef]

34. Budak, H.; Pehlivan, E. Some inequalities for weighted area balance via functions of bounded variation. Rocky Mt. J. Math. 2020, 50, 455-466. [CrossRef]

35. Budak, H.; Sarikaya, M.Z. On generalization of Dragomir's inequalities. Turkish J. Anal. Number Theory 2017, 5, 191-196. [CrossRef]

36. Cerone, P.; Dragomir, S.S.; Pearce, C.E.M. A generalized trapezoid inequality for functions of bounded variation. Turk. J. Math. 2000, 24, 147-163.

37. Dragomir, S.S. The Ostrowski integral inequality for mappings of bounded variation. Bull. Aust. Math. Soc. 1999, 60, 495-508. [CrossRef]

38. Dragomir, S.S. A companion of Ostrowski's inequality for functions of bounded variation and applications. Int. J. Nonlinear Anal. Appl. 2014, 5, 89-97.

39. Tseng, K.-L.; Yang, G.-S.; Dragomir, S.S. Generalizations of weighted trapezoidal inequality for mappings of bounded variation and their applications. Math. Comput. Model. 2004, 40, 77-84. [CrossRef]

40. Tseng, K.-L.; Hwang, S.-R.; Dragomir, S.S. Generalizations of weighted Ostrowski type inequalities for mappings of bounded variation and applications. Comput. Math. Appl. 2008, 55, 1785-1793. [CrossRef]

41. Tseng, K.-L.; Hwang, S.-R.; Yang, G.-S.; Chu, Y.-M. Improvements of the Ostrowski integral inequality for mappings of bounded variation I. Appl. Comput. Math. 2010, 217, 2348-2355. [CrossRef]

42. Tseng, K.-L.; Hwang, S.-R.; Yang, G.-S.; Chu, Y.-M. Weighted Ostrowski integral inequality for mappings of bounded variation. Taiwan. J. Math. 2011, 15, 573-585. [CrossRef]

43. Jawarneh, Y.; Noorani, M.S.M. Inequalities of Ostrowski and Simpson type for mappings of two variables with bounded variation and applications. TJMM 2011, 3, 81-94, Erratum in 2018, 10, 71-74.

44. Moricz, F. Order of magnitude of double Fourier coefficients of functions of bounded variation. Analysis 2002, 22, 335-345. [CrossRef]

45. Budak, H.; Sarikaya, M.Z. On weighted generalization of trapezoid inequalities for functions of two variables with bounded variation. Kragujev. J. Math. 2019, 43, 109-122.

46. Budak, H.; Sarikaya, M.Z. A companion of Ostrowski type inequalities for functions of two variables with bounded variation. J. Adv. Math. Stud. 2015, 8, 170-184.

47. Budak, H.; Sarikaya, M.Z. A companion of generalization of Ostrowski type inequalities for functions of two variables with bounded variation. Appl. Comput. Math. 2016, 15, 297-312.

48. Schumacher, C.S. Closer and Closer: Introducing Real Analysis, 1st ed.; Jones and Bartlett Publishers Inc.: Boston, MA, USA, 2008.

49. Clarkson, J.A.; Adams, C.R. On definitions of bounded variation for functions of two variables. Bull. Am. Math. Soc. 1933, 35, 824-854. [CrossRef]

50. Clarkson, J.A. On double Riemann-Stieltjes integrals. Bull. Am. Math. Soc. 1933, 39, 929-936. [CrossRef]

51. Samko, S.G.; Kilbas, A.A.; Marichev, I.O. Fractional Integrals and Derivatives: Theory and Applications; Gordon \& Breach: Yverdon, Switzerland, 1993.

52. Kilbas, A.A.; Srivastava, H.M.; Trujillo, J.J. Theory and Applications of Fractional Differential Equations; North-Holland Mathematics Studies, 204; Elsevier Science B.V.: Amsterdam, The Netherlands, 2006.

53. Budak, H.; Agarwal, P. On Hermite-Hadamard Type Inequalities for Co-Ordinated Convex Mappings Utilizing Generalized Fractional Integrals; Accepted as an Book Chaper, Fractional Differentiation and its Applications; Springer: Berlin/Heidelberg, Germany, 24 November 2019 